\title{
Die Deponie in einer ökologisch orientierten Volkswirtschaft
}

\author{
Peter Baccini*, Hasan Belevi und Thomas Lichtensteiger
}

Wo und wie sollen feste Rückstände aus dem Stoffwechsel einer Volkswirtschaft deponiert werden? Diese Frage scheint vordergründig an Ingenieure und Naturwissenschafter gerichtet zu sein, welche die notwendigen Qualitätskriterien und die geeigneten bau- und verfahrenstechnischen Unterlagen liefern sollen. Sie kann aber nur dann gewissenhaft beantwortet werden, wenn auch der gesellschaftspolitische Hintergrund berücksichtigt wird. Deponierungsstrategien stützen sich, bewußt oder unbewußt, auf ethisch begründete Forderungen. Will eine Gesellschaft ihren Stoffhaushalt nach ökologischen Kriterien gestalten, so muß sie sich auch entscheiden, welche festen Rückstände sie zukünftigen Generationen zur weiteren Behandlung übergeben will und welche Abfälle sie in die natürlichen Stoffkreisläufe überführt.

\begin{abstract}
Landfill strategies of a society are based, consciously or unconsciously, on ethical demands. An ecologically orientated economy is challenged to control its metabolism according to the principle of precaution. With regard to landfilling of solid wastes, the principle of precaution can be interpreted as follows: The material should have properties similar to those of the earth crust, that is natural sediments, rocks, minerals or soils. Each generation should handle its waste in a way that these properties are reached within the producers lifespan. In industrialized and affluent societies the landfills of the youngest past do not meet these criteria. They are biological and chemical reactors which have to be controlled at present and by future generations, most probably at least several hundreds of years to prevent detrimental emissions to the biosphere. The most problematic substances are the organic components which endanger the groundwater quality. With regard to their resource potential these heterogeneous mixtures of a wide variety of substances (for example municipal solid waste, industrial waste) are of minor or no value. It becomes evident that such landfill types are in contradiction to the principle of precaution. An ecologically orientated landfill strategy should be based on models of modern geology. Landfills should be understood as anthropogenic sediments which undergo diagenetic processes. The recorded history of different geogenic sediments in geological
\end{abstract}

\footnotetext{
* Postadresse : Prof. Dr. P. Baccini Abteilung Abfallwirtschaft und Stoffhaushalt Eidgenössischc Anstalt für Wasserversorgung, Abwasserreinigung und Gewässerschutz (EAWAG)

CH-8600 Dübendorf (Schweiz)
}

times gives informations on their physical and chemical transformations under different boundary conditions. From this one can deduce quality criteria for anthropogenic sediments. A first evaluation of fwo products of waste treatment plants, namely bottom ashes and filter dust from incineration of municipal solid waste, shows that these criteria are not yet met. It follows that on a short-term range a strong improvement of process engineering and a capacity increase of waste treatment plants (mainly separate collection and thermal treatment) is indispensable. However, projects of new waste treatment plants in densely populated regions are very often rejected by the people of the community which is selected. On a long-term range only an adaptation of the whole economy to the above mentioned criteria is ecologically reasonable. Such an adaptation can not be achieved solely by technical means. It touches severely the existing hierarchy of values of a national economy. Therefore it is necessary to combine the methods of political and social sciences with those of the natural and engineering sciences to transpose landfill strategies into practice.

\section{Einleitung}

Zur Charakteristik von Überflußgesellschaften gehören steigende Güterflüsse in der Versorgung und steigende Abfallflüsse in der Entsorgung 1"I. Das mengenmäßig wichtigste Gut ist Wasser, gefolgt von Luft. Beide verlassen die anthropogenen Prozesse (private und öffentliche Haushalte, Güterproduktion und Entsorgung) meistens unmittelbar nach Gebrauch, allerdings in veränderter chemischer Zusammensetzung, in Richtung Hydrosphäre und Atmosphäre. Bezüglich dieser beiden Güter befindet sich die Anthroposphäre nahezu in einem Fließgleichgewicht. Der Fluß der festen Materialien, mengenmäßig dominiert durch die Baustoffe (hauptsächlich $\mathrm{Ge}$ steine), ist auf der Inputseite größer als auf der Outputseite. Die bauliche Infrastruktur wächst, das heißt, die Menge der Gebäude und deren Ausrüstung sowie Verbindungswege (in erster Linie Straßen) nimmt zu, und zwar pro Einwohner ${ }^{|2|}$. In Regionen wie Westeuropa, Nordamerika und Japan betrug diese Menge zu Beginn der neunziger Jahre schätzungsweise 200-300 Tonnen pro Einwohner "I. Vergleicht man den ökonomischen Summenparameter der volkswirtschaftlichen Aktivität, das Bruttosozialprodukt, mit den Güterflüssen, so stellt man fest, daB die relativen Wachstumsraten etwa gleich sind, nämlich einige Prozent pro Jahr ${ }^{121}$.

Auf der Entsorgungsseite manifestiert sich der Fluß fester Güter noch nicht in gleicher Weise, weil die in den letzten Jahrzehnten rasch gewachsene Infrastruktur noch relativ jung ist und sich die Prozesse der Substitution und Erneuerung erst in den nächsten Jahren mengenmäßßig immer stärker auswirken ${ }^{[3 \mid}$. Einer Menge von 5-10 Tonnen fester Güter pro Einwohner und Jahr in der Versorgung stehen etwa 1-3 Tonnen in der Entsorgung gegenüber. In allen Überflußgesellschaften wurde bis heute der größte Teil der festen Abfälle, mit oder ohne Vorbehandlung, deponiert. Auch bei einer starken Erhöhung der Recyclingquoten wird sich zumindest im nächsten Jahrzehnt die zu deponierende Menge nicht wesentlich vermindern. Für die Schweiz zum Beispiel rechnet man bei optimalem Vollzug aller vorgesehenen Maßnahmen mit einer mengenmäßigen Reduktion von einem Viertel in einer Zeitspanne von etwa zehn Jahren ${ }^{14}$. Noch dazu ist aus naturgesetzlichen Gründen ein vollständiges Recycling der aus der Anthroposphäre stammenden festen Güter ohne sehr große Betriebsgüterflüsse (Energieträger) nicht möglich. Die Deponie als anthropogener Prozeß bleibt somit essentieller Teil jeder Volkswirtschaft. Quantität und Qualität der zu deponierenden Feststoffe sind insgesamt abhängig von der kulturellen Eigenart einer Gesellschaft, also von der Summe ihrer ethischen, politischen, wirtschaftlichen und wissenschaftlich-technischen Eigenschaften.

Die Geschichte der Deponien ist eng verknüpft mit der Entwicklung der 
Städte. Bereits in der Antike kannte man die durch Ablagerung fester Abfälle hervorgerufenen Widerstände jener meist armen Bevölkerungsteile, die an der Peripherie von Städten inmitten von Deponien zu leben hatten. Eine Deponie bedeutete Geruchsbelästigung und ästhetisches Ärgernis ${ }^{15}$. Erst im 19. Jahrhundert wurde die Deponie von Siedlungsabfällen aus wissenschaftlicher Sicht als Hygieneproblem erkannt. In der zweiten Hälfte des 20. Jahrhunderts, mit dem Auftreten großräumiger Überflußgesellschaften wurde deutlich, daß stofflich sehr komplex zusammenge-

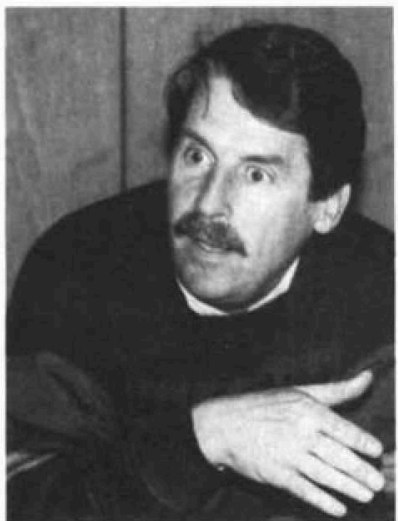

Peter Baccini: Geboren 1939 in Zürich; Studium der $\mathrm{Na}$ turwissenschaften an der Eidgenössischen Technischen Hochschule (ETH) Zürich; Promotion 1968. Fünfjährige Tätigkeit in Forschung und Entwicklung der chemischen Industrie in Basel. Seit 1974 an der Eidgenössischen Anstalt für Waserversorgung, Abwasserreinigung und Gewässerschutz (EAWAG) und Professeur associé en chimie de l'environnement an der Universităt Neuchâtel. Wissenschaftliche Arbeiten über den Stoffhaushalt von Seen. Seit 1983 Leiter der Abteilung Abfallwirtschaft und Stoffhaushalt der EAWAG. Interdisziplinäre Forschungsarbeiten (Natur- und Ingenieurwissenschaften, Ökonomie) über den regionalen Stoffhaushalt, die resultierenden Umweltbelastungen und Entsorgungsstrategien. Seit 1991 Professor für Stoffhaushalt und Entsorgungstechnik an der ETH Zürich.

Hasan Belevi: Geboren 1953. Chemie-IngenieurStudium an der ETH Zürich; Promotion 1980. Vierjährige Tätigkeit in Forschung und Entwicklung der chemischen Industrie in Zürich und Visp. Seit 1985 an der EAWAG. Gastwissenschafter an der Stanford University, USA (1988-1989). Seit 1990 Lehrbeauftragter an der ETH Zürich. Forschungsarbeiten über die "chemische Selektivität bei den durch molekulare Diffusion maskierten Reaktionen" (1976-1981) und das "Langzeitverhalten der Reaktordeponien".

Thomas Lichtensteiger: Geboren 1956. Studium der Erdwissenschaften an der Universität Basel; Promotion 1986. Einjährige Berufserfahrung in Reservoir-Geologie (Erdöl). Seit 1986 an der EAWAG. Forschungsarbeiten über anthropogene Sedimente (Klärschlamm, Schlacke aus Kehrrichtverbrennungsanlagen). Seit 1990 Leiter der Gruppe Entsorgung in der Abteilung Abfallwirtschaft und Stoffhaushalt der EAWAG. Seit 1991 Lehrbeauftragter an der ETH Zürich. setzte Ablagerungen zur Quelle von Emissionen werden können, welche vor allem die natürlichen Trinkwasserressourcen gefährden. Die jüngste Geschichte anthropogener Ablagerungen führte somit zu zwei neuen Aktivitäten, nämlich einerseits wasserverschmutzende Deponien zu sanieren ("Altlastensanierung") und anderseits potentielle Emittenten zu eruieren ("Altlastenerfassung", eine Art Spurensicherung rezenter Sedimente). Die in den letzten Jahrzehnten entwickelten staatlichen Vorschriften zur Regelung der Deponierung stützen sich denn auch primär auf die Gewässerschutzgesetze. Die Deponie wird in diesem Zusammenhang zunächst als ein geotechnisches Bauwerk betrachtet, das mit einer Kombination von Dichtungen ausgerüstet sein muß, um Emissionen zu vermeiden ${ }^{|6|}$. Darüber hinaus hat die Deponie aber auch als biogeochemischer Reaktor eine Langzeitwirkung, weil im Falle von Siedlungsabfällen die ökologisch relevante Laufzeit Jahrhunderte dauern kann ${ }^{17}$.

In den achtziger Jahren orientierten sich die Entsorgungskonzepte der Überflußgesellschaften immer stärker an ethisch begründeten ökologischen Grundsätzen, die auch in den Präambeln der neu geschaffenen Umweltschutzgesetze verankert wurden (zum Beispiel das Vorsorgeprinzip). In einem ethischen Diskurs sollte ein gesellschaftspolitischer Konsens erreicht werden, der ein tragfähiges Fundament für die weiteren Entscheidungen bei der konkreten Ausgestaltung der Abfallwirtschaft bildet. Die Deponie spielt dabei eine Schlüsselrolle. Es ist daher evident, da $\beta$ die künftig einzuschlagende Deponierungsstrategie primär eine Funktion der ethischen Grundsätze darstellt, zu welchen sich eine Mehrheit einer demokratischen $\mathrm{Ge}$ sellschaft bekennen will. Eine kürzlich publizierte Synopsis zeigt, daß Deutschland, Österreich und die Schweiz ihre Deponierungskonzepte auf vergleichbaren Grundsätzen aufbauen wollen ${ }^{\text {[8] }}$.

Unser Übersichtsartikel hat zum Ziel, am Beispiel einer dicht besiedelten $\mathrm{Re}$ gion die Konsequenzen einer ökologisch orientierten Volkswirtschaft für die Deponierung von festen anthropogenen Reststoffen zu skizzieren. Insbesondere sollen folgende Fragen beantwortet werden:

- Welche quantitative und qualitative Bedeutung haben die schon vorhandenen Deponien, unter denen sich auch die sogenannten Alllasten befinden, für die zukünftige Ressourcenbewirtschaftung und die Umweltschutzstrategie der Region?
- Welche Konsequenzen ergeben sich für den Bau und den Betrieb der neu zu schaffenden Deponien und darüber hinaus für den ganzen Stoffhaushalt dieser Volkswirtschaft?

\section{Grundsätze und Entscheidungskriterien}

Die Wahl der Deponierungsstrategien stützt sich auf gesellschaftspolitische Entscheidungen, die ihrerseits als Ergebnis eines ethischen Diskurses begriffen werden müssen. Daher kann eine Darstellung der Deponie in einer Volkswirtschaft nicht ohne diese Basis auskommen. Es soll deshalb zuerst dargelegt werden, was unter einer ökologisch orientierten Volkswirtschaft im Falle der Abfallwirtschaft verstanden wird. Anschließend ist ein für dicht besiedelte Regionen mit Überflußgesellschaften repräsentativer Rahmen zu wählen, innerhalb dessen die in der Einleitung gestellten Fragen qualitativ und quantitativ diskutiert werden können.

\subsection{Ethische Grundsätze zur Deponierung} am Beispiel des schweizerischen Leitbildes für die Abfallwirtschaft 191

Das Leitbild stützt sich einerseits auf den Zweckartikel des 1985 in Kraft gesetzten Umweltschutzgesetzes, in dem das Vorsorgeprinzip und das Verursacherprinzip verankert sind, anderseits auf einen in den achtziger Jahren durchgeführten politischen Diskurs zur Ausrichtung der Entsorgung. Die folgenden vier Leitsätze widerspiegeln den in dieser Zeit erreichten politischen Konsens zwischen den wichtigsten Interessengruppen:

(I) Die Entsorgung hat vor allem und als Ganzes umweltverträglich zu sein.

Für die Abfallwirtschaft als Zweig der Volkswirtschaft gilt somit, daß der (auch langfristige) Schutz des Menschen und der ganzen Biosphäre vor der Wirtschaftlichkeit zu kommen hat. Dabei wird die Tatsache berücksichtigt, daß bis heute eine Internalisierung der externen Kosten noch nicht verwirklicht ist. Allerdings kann sich vorerst nur eine relativ reiche Gesellschaft eine solche Wertordnung leisten. Es genügt auch nicht, daß nur einzelne Teile (zum Beispiel die Verbrennung) eines Entsorgungssystems die Umweltverträglichkeitsprüfung bestehen (zum Beispiel bezüglich Luft und Wasser). Nicht zu- 
letzt müssen die festen Rückstände in einen Zustand gebracht werden, der späteren Generationen keine "Altlasten" beschert. Mit anderen Worten: Jede Generation löst ihre Entsorgungsprobleme möglichst vollständig, das heißt finanziell und technisch. Die öffentliche Hand wattet dabei nur subsidiär.

(2) Die Schweiz entsorgt möglichst vollständig im eigenen Land mit maßgeschneiderten Lösungen für die unterschiedlichen Regionen.

Es ist ethisch unzulässig, Abfälle mit hohem Schadstoffpotential in Regionen zu transferieren, die nicht über nach unseren Maßstäben geeignete Behandlungsanlagen verfügen. Weil Quantität und Qualität der Abfälle stark von der wirtschaftlichen Struktur einer Region abhängen, gibt es keine universell anwendbaren Entsorgungssysteme. In Übereinstimmung mit Leitsatz (1) muß die Deponieplanung daher langfristig entworfen, also auch ein essentieller Teil der Raumplanung werden. Daraus lassen sich zwei naturwissenschaftlichtechmische Zielsetzungen für die Entsorgung, speziell für die Deponierung herleiten:

(3) Entsorgungssysteme produzieren aus Abfällen nur zwei Arten von Stoffklassen, nämlich wiederverwertbare Stoffe und endlagerfähige Reststoffe.

Endlagerfähig ist ein Reststoff dann, wenn er ohne oder mit einer Hülle (nach geochemischen und geophysikalischen Kriterien ausgewählt) langfristig (über Hunderte von Jahren) nur solche Stoffe an Luft, Wasser und Boden abgibt, welche diese in ihren chemischen und physikalischen Eigenschaften nicht signifikant verändern. Die heute betriebenen Siedlungsabfalldeponien sind demnach keine Endlager, sondern Reaktordeponien, die als Behandlungsverfahren während Jahrzehnten, oft sogar Jahrhunderten vom Menschen kontrolliert werden müssen ${ }^{171}$.

Aufgrund heutiger Kenntnisse kann verlangt werden, daß organische Stoffe nicht in ein Endlager gehören, da sie früher oder später von Mikroorganismen als Energie- oder Nährstoffquelle benutzt werden. Die dabei entstehenden Metaboliten (im Deponiegas oder im Sickerwasser) sind potentielle Schadstoffe für die benachbarten Kompartimente. Biogene Abfälle sollten deshalb in möglichst reiner Form wiederverwertet werden (zum Beispiel als bodenverbessernder Kompost, das heißt als ein bodenähnlicher Stoff). Xenobiotische organische Stoffe hingegen sollten, falls nicht wiederverwertbar, vollständig mineralisiert werden.

(4) Abfallbehandlungsverfahren müssen so konzipiert werden, daß aus den zu deponierenden festen Rückständen erdkrustenähnliche, alsoboden-, gesteinsoder erzähnliche Stoffe entstehen.

Komplementär zum Leitsatz (3) soll auch ein langfristiges Ziel für die Ressourcenbewirtschaftung gesetzt werden. Es genügt nicht, nur unmittelbar wiederverwertbare Stoffe aus Abfällen zu gewinnen; auch die abzulagernden festen Reststoffe sollen qualitativ so beschaffen sein, daß zukünftige Generationen sie eventuell als neue Ressourcen nutzen könnten. Diese Zielsetzung hat zur Konsequenz, daß keine Gemische von Stoffen mit sehr unterschiedlichen Eigenschaften deponiert werden (in der Fachliteratur als "co-disposals" bezeichnet), wie das heute noch in vielen Ländern geschieht, so beispielsweise Gemische von Schlacken und Filterstäuben aus Kehrichtverbrenungsanlagen oder von Betonabbruch und Backsteinen. Notwendig werden Monodeponien ("monofills"), das heißt Deponien mit nur einer chemisch möglichst gut definierten Stoffsorte. Monodeponien, welche noch keine Endlagerqualität aufweisen und somit als Reaktordeponien geführt werden müssen, sind immerhin einfacher zu kontrollieren und bieten bessere Chancen, in kürzerer Frist (einige Jahrzehnte) Endlagerqualität zu erreichen.

Zusammenfassend sei hervorgehoben daß die Leitsätze (3) und (4) als Folge der ethisch begründeten Leitsätze (I) und (2) $z u$ verstehen sind. Mit naturwissenschaftlichen Argumenten allein läßt sich das Endlagerkonzept nicht durchsetzen ${ }^{10 !}$. Es erscheint durchaus möglich, eine umweltverträgliche Reaktordeponie über Jahrhunderte zu betreiben. Auch das Ziel Ressourcenschonung und/oder Schaffung potentieller Vorräte für zukünftige Generationen setzt auf das Prinzip Verantwortung "'I. Der im Titel unseres Beitrags gewählte Begriff "ökologisch orientierte Volkswirtschaft" will in diesem Sinne verstanden werden.

\subsection{Beschreibung einer fiktiven Region "Metaland" zur Illustration der Deponierung fester Abfälle}

In Tabelle 1 sind für eine Region, welche als repräsentativ für die Situation einer dicht besiedelten Region in Westeuropa gelten kann, einige wichtige und für diese Studie notwendige Kenndaten zusammengestellt. Es handelt sich um eine Region, deren Anthroposphäre einen Stoffwechselprozeß von sogenannten Überflußgesellschaften zeigt "II. Das Ent-

Tabelle 1. Eigenschaften und Kenndaten der Region "Metaland" [1]

\begin{tabular}{|c|c|c|}
\hline Eigenschaften & Kenndaten & Bemerkungen \\
\hline Fläche & $2500 \mathrm{~km}^{2}$ & \\
\hline $\begin{array}{l}\text { Bevölkerung } \\
\text { Dichte }\end{array}$ & $\begin{array}{l}1000000 \text { Einwohner }(E) \\
400 \mathrm{E} / \mathrm{km}^{2}\end{array}$ & \\
\hline \multicolumn{3}{|l|}{ Flächennutzung } \\
\hline $\begin{array}{l}\text { Landwirtschaft } \\
\text { Forstwirtschaft } \\
\text { Oberflächengewässer } \\
\text { und unproduktives Land }\end{array}$ & $\begin{array}{r}55 \% \\
30 \% \\
3 \%\end{array}$ & \\
\hline \multicolumn{3}{|l|}{ Hydrologie } \\
\hline Nettoniederschlag & $0.9 \times 10^{9} \mathrm{~m}^{3} / \mathrm{a}$ & $\begin{array}{l}\text { Niederschlag minus Evaporation } \\
0.35 \mathrm{~m}^{3} / \mathrm{m}^{2} \cdot \mathrm{a}\end{array}$ \\
\hline Oberflächenzufluß & $1.0 \times 10^{9} \mathrm{~m}^{3 / a} \mathrm{a}$ & \\
\hline Seevolumen & $2.8 \times 10^{9} \mathrm{~m}^{3}$ & $\begin{array}{l}\text { Fläche: } 56 \mathrm{~km}^{2} \\
\text { mittlereTiefe: } 60 \mathrm{~m}\end{array}$ \\
\hline Grundwasserreservoir & $2.0 \times 10^{9} \mathrm{~m}^{3}$ & isolierte Grundwasserlinse \\
\hline \multicolumn{3}{|l|}{ Anthropogener Stoffwechsel } \\
\hline Wasser/Abwasser & $90 \mathrm{~m}^{3} / \mathrm{E} \cdot \mathrm{a}$ & \\
\hline $\begin{array}{l}\text { Luft/Abluft } \\
\text { Feststoffe }\end{array}$ & $20 \times 10^{3} \mathrm{~kg} / \mathrm{E} \cdot \mathrm{a}$ & inklusive flüssige fossile Brennstoffe \\
\hline $\begin{array}{l}\text { Input } \\
\text { Output } \\
\text { Lager }\end{array}$ & $\begin{array}{l}9.0 \times 10^{3} \mathrm{~kg} / \mathrm{E} \cdot \mathrm{a} \\
2.5 \times 10^{3} \mathrm{~kg} / \mathrm{E} \cdot \mathrm{a} \\
2.6 \times 10^{5} \mathrm{~kg} / \mathrm{E}\end{array}$ & \\
\hline \multicolumn{3}{|l|}{ Entsorgungsinfrastruktur } \\
\hline Deponie & $4.0 \times 10^{5} \mathrm{~kg} / \mathrm{E}$ & $\begin{array}{l}\text { Reaktordeponien, ca. } \\
1 \text { pro } 1000 \text { Einwohner }\end{array}$ \\
\hline Verbrennungsanlagen & 5 & $\begin{array}{l}1 \text { pro } 200000 \text { Einwohner, } \\
\text { mit Kapazität von ca. } 10^{4} \mathrm{~kg} / \mathrm{h}\end{array}$ \\
\hline
\end{tabular}


sorgungssystem ist technisch gut ausgebaut, das heißt, mehr als 90 Prozent der Einwohner (Wohn- und Arbeitsplätze) sind

- an ein Kanalisationssystem mit Abwasserreinigungsanlage angeschlossen;

- zur Entsorgung von festen Abfällen durch Sammelorganisationen an Verbrennungsanlagen und/oder Sortieranlagen und Deponien angeschlossen. Die angegebene Deponiemenge widerspiegelt die stoffliche Entsorgungsgeschichte der Region "Metaland". Die Zahlenangaben basieren auch auf Altlastenerhebungen des Kantons Aargau ${ }^{[12 !}$.

\section{Ressourcen- und Belastungs- potential bestehender Deponien}

\subsection{Ressourcen- und Schadstoffpotentiale von Abłällen}

Die in "Metaland" entstehenden Abfälle werden in fünf Kategorien eingeteilt. Die in Tabelle 2 angegebenen Mengen entsprechen jenen des schweizerischen Durchschnitts für die achtziger Jahre ${ }^{14]}$. Durch seinen hohen Wassergehalt von circa $94 \%$ ist der Klärschlamm zur Zeit die mengenmäßig wichtigste Kategorie. Ungefähr $30 \%$ des Klärschlamms werden deponiert. Mit dem Ausbau der Kläranlagen (Figur I) hat die Erzeugung von Klärschlamm steil zugenommen, in der Schweiz innert dreißig Jahren auf mehr als das Zehnfache ${ }^{1131}$. Die Siedlungsabfälle machen rund ein Drittel der gesamten Abfallproduktion aus, pro Kopf hat sich der Siedlungsabfall während der letzten Generationsdauer verdoppelt ${ }^{|14|}$. Noch vor einer Generation betrug der Verbrennungsanteil weniger als $20 \%$ (Figur 1). In den achtziger Jahren wurden $80 \%$ des Siedlungsabfalls verbrannt, der Rest deponiert. Die Rückstände der Verbrennung (Schlacke und Rückstände der Rauchgasreinigung) wurden meist vermischt deponiert oder im Straßenbau wieder verwendet ${ }^{115\}}$. Die Abfälle aus der Bautätigkeit, hier als Bausperrgut bezeichnet ${ }^{|16|}$, sind mengenmäßig gleich einzuordnen wie die Siedlungsabfälle. Sie wurden in der Vergangenheit mit wenigen Ausnahmen deponiert. Ihre mengenmäßige Entwicklung als Funktion der Zeit ist nicht genügend dokumentiert; es wird angenommen, daß die Wachstumsrate derjenigen der Siedlungsabfälle gleicht. Nicht berücksichtigt sind hierbei die anderen Baurestmassen, nämlich Aushub, Straßenaufbruch,
Tabelle 2. Abfallkategorien.

\begin{tabular}{lccrr}
\hline Kategorie & \multicolumn{2}{c}{ Abfallflux } & \multicolumn{2}{c}{ Deponierte Menge } \\
& {$\left[\mathrm{kg} \mathrm{E}^{-1} \mathrm{a}^{-1}\right]$} & {$[\%]$} & 200 & 30 \\
\hline Klärschlamm a) & 660 & 41 & 90 & 20 \\
Siedlungsabfälle & 440 & 28 & 460 & 100 \\
Bauabfälle b) & 460 & 29 & 20 & 100 \\
Shredder- und Pneuabfälle & 20 & 1 & 10 & 50 \\
Sonderabfälle & 20 & 1 & 780 & 50 \\
Total & 1600 & 100 & $\left.\mathrm{~kg}^{-1}\right]$ & {$[\%]$}
\end{tabular}

a) $94 \%$ Wasser. b) Inklusive direkt deponierte Industrieabfälle (nach Fahrni ${ }^{(4)}$ )

Tabelle 3. Durchschnittliche Fluxe ausgewählter Elemente in Abfällen.

\begin{tabular}{|c|c|c|c|c|c|}
\hline \multirow[t]{2}{*}{ Abfallkategorie } & \multicolumn{5}{|c|}{$\begin{array}{l}\text { Elementflux in Abfällen } \\
\qquad\left[\mathrm{kg} \mathrm{E}^{-1} \mathrm{a}^{-1]}\right.\end{array}$} \\
\hline & $\mathrm{C}_{\text {org. }}$ & $\mathrm{Cl}$ & $\mathrm{Zn}$ & $\mathrm{Cd}$ & $\mathrm{Hg}$ \\
\hline Klärschlamm & 7 & 0.1 & 0.1 & 0.001 & 0.0004 \\
\hline Siedlungsabfälle & 130 & 3.0 & 0.5 & 0.006 & 0.0010 \\
\hline Bauabtälle & 55 & 0.4 & 0.2 & 0.002 & 0.0000 \\
\hline Shredder- und Pneuabtälle & 12 & 0.5 & 0.7 & 0.001 & 0.0000 \\
\hline Sonderabfälle & 10 & 0.5 & 0.1 & 0.000 & 0.0004 \\
\hline Total & 210 & 5 & 2 & 0.01 & 0.0020 \\
\hline
\end{tabular}

Mischabbruch und Asphaltbeton [17], welche teilweise oder vollständig wieder im Baugewerbe verwendet wurden. Für diese Betrachtung wird angenommen, daß Ablagerungen solcher Rückstände nicht zu den im folgenden aufgeführten Altlasten zählen. Allerdings fehlen dazu experimentelle Untersuchungen. Als Shredder- und Pneuabfälle bezeichnet man die nichtmetallischen Rückstände aus der Aufbereitung von Altautos sowie anderen überwiegend aus Eisen und Stahl bestehenden Gütern wie ausgedienten industriellen Apparaten, Kühlschränken und Waschmaschinen. Diese Abfälle, auch als RESH bezeichnet ${ }^{181}$, wurden hauptsächlich deponiert. Die Sonderabfälle, eingestuft gemäß Verordnung über den Verkehr mit Sonderabfällen in der Schweiz (VVS) ${ }^{|19|}$, erreichen die gleiche Größenordnung wie die RESH-Abfälle, beide zusammen stellen jedoch nur einige Prozent des gesamten Abfalls. Über ihre zeitliche Entwicklung liegen keine verläßlichen Daten vor. Für die Sonderabfälle wird angenommen, daß in der Vergangenheit eine Hälfte deponiert, die andere verbrannt wurde.

Zur Beurteilung des Ressourcen- oder Schadstoffpotentials sind Kenntnisse über die chemische Zusammensetzung der in Tabelle 2 genannten Abfälle nötig. Mangels hinreichender Daten sind wir noch angewiesen auf wenige, relativ junge Untersuchungen, die sich auf wenige Indikatorelemente (Tabelle 3) kon-

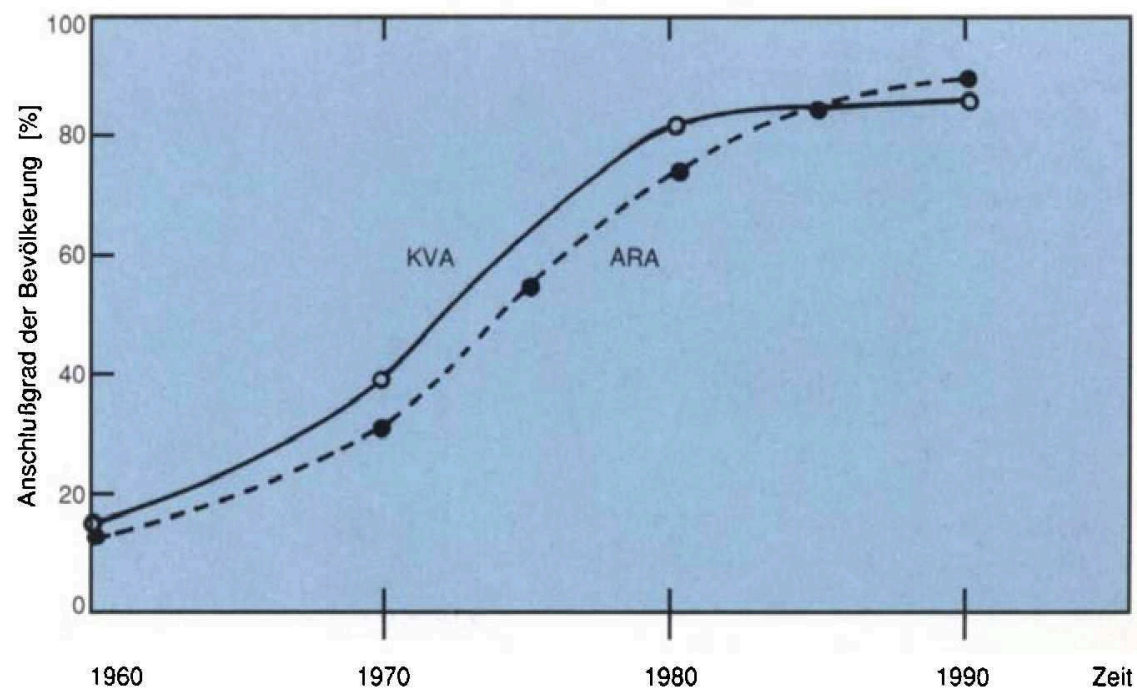

Figur 1. Die Zunahme der Abwasser- und Abfallbehandlungsanlagen in der Schweiz während der letzten drei Jahrzehnte. KVA: Kehrichtverbrennungsanlagen; ARA: Abwasserreinigungsanlagen [13]. 
zentrieren ${ }^{|20|}$. Tabelle 3 zeigt die Fluxe von fünf Indikatorelementen durch die fünf Abfallkategorien. Der organische Kohlenstoff steht für die Summe der organischen Verbindungen, Chlor für die Halogene (einerseits als hydrophiles Chlorid, anderseits in den halogenierten organischen Verbindungen). Zink ist sowohl ein essentielles Element als auch ein von Menschen breit verwendetes Metall. Cadmium und Quecksilber sind Vertreter von Elementen mit einem relativ hohen Schadstoffpotential. Die Fluxe aller drei Metalle im Gesamtabfall haben die gleiche Größenordnung wie der jährliche Konsum ${ }^{1201}$. Insgesamt betrachtet sind die Siedlungsabfälle der Hauptträger dieser Elemente mit mehr als $60 \%$ der totalen Elementfluxe. Für Zink findet man auch einen großen Flux durch die Shredder- und Pneuabfälle. Sonder- und Shredderabfälle können ein hohes lokales Schadstoffpotential darstellen. Regional betrachtet haben jedoch die Siedlungsabfälle das höchste Ressourcen- und Schadstoffpotential.

Wenn es gelingt, die brauchbaren Stoffe in den Abfällen wieder zu nutzen, können Rohstoffe gespart werden. Für eine Wiederverwertung müssen die Stoffe jedoch relativ rein und konzentriert vorkommen, so daß sie sich in einem technischen Prozeß mit nicht sehr hohem Energieaufwand separieren, aufkonzentrieren und veredeln lassen. Damit dieser Prozeß unter gegenwärtigen Marktbedingungen wirtschaftlich werden kann, ist mindestens erforderlich, daß die Konzentrationen in der gleichen Größenordnung sind wie bei den natürlich vorkommenden Erzen.

Die Konzentrationen der in Tabelle 4 angegebenen Indikatorelemente $\mathrm{Zn}, \mathrm{Cd}$ und $\mathrm{Hg}$ im Klärschlamm sind mindestens un zwei Größenordnungen niedriger als diejenigen in den Erzen. Auch im getrockneten Klärschlamm wären sie immer noch zu tief für eine Aufbereitung als Erz. Hingegen reicht der Gehalt an organischem Kohlenstoff für eine Verwendung als Brennstoff oder für die Kompostierung aus.

Die anderen Abfallkategorien enthalten große Anteile organischen Materials. Innerhalb einer Abfallkategorie kommt jedoch organischer Kohlenstoff in einer großen Vielfalt von Verbindungen vor und kann deshalb meistens nicht als rein betrachtet werden. Eine technische Separierung und Veredelung kommt höchstens bei Shredder- und Sonderabfällen in Frage. Ein wirtschaftlich günstigeres Verfahren ist bei den meisten Abfällen das separate Einsammeln zum Zweck einer Wiederverwendung. Für

Siedlungs- und Bauabfälle ist dies zur Zeit der einzige gangbare Weg, um organische Stoffe zu rezirkulieren ${ }^{|2|}$.

Von Chlor, Cadmium und Quecksilber sind die Konzentrationen in jeder Abfallkategorie viel niedriger als in Erzen, sie können deshalb mit technischen Prozessen nicht leicht zurückgewonnen werden. Shredderabfälle und zum Teil auch die Sonderabfälle haben aber ein gewisses Ressourcenpotential für Zink.

Im allgemeinen werden Stoffe bei ihrem Gebrauch mit anderen Stoffen vermischt und verteilt, also ökonomisch entwertet. Es würde viel Energie benötigt, um die Stoffe wieder in ihrer ursprünglichen Form an den Verwendungsort zu bringen. Wenn Abfälle der erwähnten Kategorien unbehandelt in Deponien gelangen, ist eine Wiedergewinnung mancher Stoffe ökonomisch und ökologisch nicht sinnvoll. Das

Tabelle 4. Durchschnittliche Konzentrationen ausgewählter Elemente in Abfällen und Vergleich mit dem Gehalt in Erzen und in der Erdkruste.

\begin{tabular}{lccccc}
\hline Abrallkategorie a) & \multicolumn{5}{c}{ Konzentration [g/kg] } \\
& $\mathrm{C}_{\text {org. }}$ & $\mathrm{Cl}$ & $\mathrm{Zn}$ & $\mathrm{Cd}$ & $\mathrm{Hg}$ \\
\hline Klärschlamm & 10 & 0.1 & 0.2 & 0.001 & 0.00060 \\
Siedlungsabfälle & 300 & 7 & 1.2 & 0.010 & 0.00500 \\
Bauabfälle & 120 & 0.8 & 0.5 & 0.003 & 0.00010 \\
Shredderabfällle & 600 & 22 & 37.0 & 0.050 & 0.00150 \\
Sonderabfälle & 480 & 22 & 7.0 & 0.020 & 0.02000 \\
Erz & 900 b) & $600 \mathrm{c})$ & 40.0 & 4.400 & 2.00000 \\
Erdkruste & 0.2 & 0.13 & 0.07 & 2 & 0.00008 \\
\hline
\end{tabular}

a) $94 \%$ Wasser. b) Fossile organische Verbindungen (Erdöl, Kohle). c) Kochsalz.

Ressourcenpotential von Deponien dieser Abfälle ist daher sehr gering, doch gibt es auch einige Ausnahmen wie Monodeponien für Galvanikschlämme, die zur Kategorie der Sonderabfälle gehören.

Tabelle 4 zeigt auch einen Vergleich von Konzentrationen der Indikatorelemente in Abfällen mit den durchschnittlichen Konzentrationen in der Erdkruste (vergleiche dazu Abschnitt 4). In jeder Abfallkategorie sind die Konzentrationen höher als in der Erdkruste. Sämtliche Abfälle enthalten genügend organischen Kohlenstoff, um sich als Substrat für Mikroorganismen zu eignen, wenn sie ohne Vorbehandlung deponiert werden. Die Deponien solcher Abfälle sind als Reaktordeponien zu betrachten und besitzen ein Umweltgefährdungspotential, das im folgenden (Abschnitt 3.2) quantifiziert wird. Die in den Abfällen gespeicherte Energie sollte zwar in Verbrennungsprozessen oder Reaktordeponien (methanhaltige Gase) genutzt werden, ihr potentieller Beitrag zur gesamten Energieversorgung einer Überflußgesellschaft ist jedoch marginal ${ }^{[20]}$.
Aus alledem geht hervor, daß Abfälle, welche tel quel in Deponien gelagert werden, kein ins Gewicht fallendes Ressourcenpotential für kommende $\mathrm{Ge}$ nerationen haben.

\subsection{Kurz- und Langzeitverhalten der} Reaktordeponien für Siedlungsabfälle

Als wichtiges Beispiel einer Reaktordeponie sei die Siedlungsabfalldeponie gewählt. Eine sogenannte geordnete Deponie kann als ein "chemisch-biologischer Festbettreaktor" betrachtet werden. Die Siedlungsabfälle werden schichtweise diskontinuierlich in den Reaktor (Reaktordeponie) eingeführt. $\mathrm{Zu}$ diesem heterogenen und unbeweglichen Inhalt trittt Wasser größtenteils als Niederschlag kontinuierlich in den Reaktor ein, sowohl in der Füllungsphase als auch nachher. Es 
die Gasentwicklung wird vernachlässigbar klein. Der Export der Elemente durch das Sickerwasser dauert viel länger. Das Sickerwasser soll gemäß heutigen Schutzzielen solange behandelt werden, bis die Elementfrachten die Umwelt nicht mehr negativ beeinflussen können.
Im allgemeinen werden aus der Deponie in den ersten 10 Jahren mehr Nichtmetalle exportiert als Metalle. Eine wichtige Beobachtung ist, daß nach der intensiven Reaktorphase, wenn also die Gasentwicklung vernachlässigbar klein geworden ist, sich immer noch mehr als $99.9 \%$ der Metalle und mehr
Figur 2.

Schematische

Darstellung der

Elementfrachten

aus einer

Siedlungsabfalldeponie

als Funktion der

Zeit.

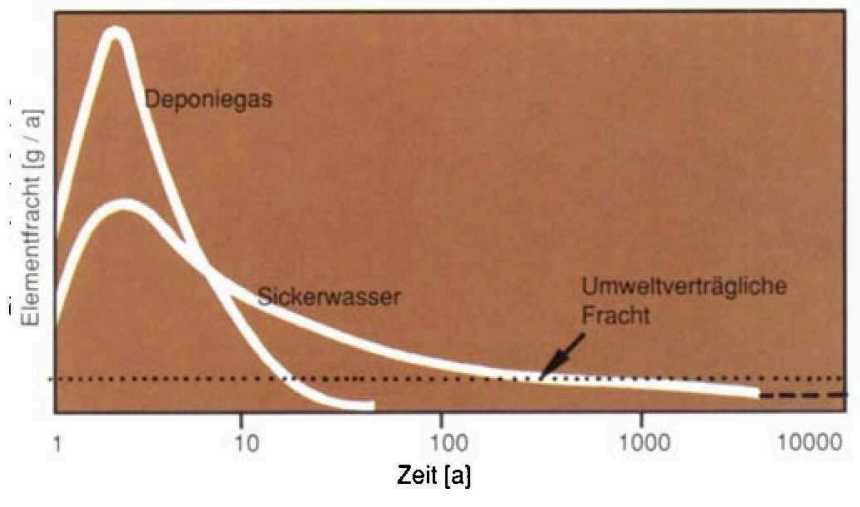

als $90 \%$ der Nichtmetalle (ausgenommen Kohlenstoff) in der Deponie befinden.

Somit haben Reaktordeponien auch nach der Phase intensiver Gasentwicklung immer noch ein hohes Schadstoffpotential. Die Emissionen aus einer älteren Deponie, bei der die Gasentwicklung auf ein vernachlässigbar niedriges Niveau gesunken ist, sind in Tabelle 5 durch Angabe der Konzentrationen im Sickerwasser und einen Vergleich mit den Anforderungen an Einleitungen in ein Gewässer ${ }^{123]}$ charakterisiert. Dieser Vergleich ist als eine erste Näherung zu verstehen, da umweltverträgliche Frachten von mehreren Faktoren abhängig sind und Emissionen mit kleineren Konzentrationen als die hier aufgeführten Grenzwerte nicht immer als umweltverträglich eingestuft werden können. Sie können jedoch nach heutiger Vollzugspraxis im Gewässerschutz als minimale Anforderungen gelten.
Das Verhalten der Deponie in der intensiven Reaktorphase kann durch Stoffflußanalysen an verschiedenen Reaktordeponien ausreichend genau charakterisiert werden ${ }^{211}$. Da die kontrollierten Siedlungsabfalldeponien (registrierte Abfallmengen, kontrollierte Ablagerung, registrierte Gas- und Sickerwasserfluisse) erst seit 20-25 Jahren existieren und es deshalb keine Erfahrungen mit älteren Deponien gibt, muß das Langzeitverhalten anhand von Modellen abgeschätzt werden.

Figur 3 zeigt die Transferkoeffizienten für elf Indikatorelemente. Die Transferkoeffizienten $k_{\mathrm{i}, j}(t)$ werden wie folgt definiert ${ }^{\mid 221}$

\section{$k_{\mathrm{ij}}(t)=$}

(Output des Elements i durch den Fluß $\mathrm{j}$ bis zur Zeit $t$ ) (totaler Input des Elements i bis zur Zeit $\iota$ )

$k_{\mathrm{Cl}, \mathrm{w}}(10)=0.05$ heißt zum Beispiel, daß $5 \%$ der anfänglichen Chlormenge in der Deponie durch das Sickerwasser in den ersten 10 Jahren exportiert werden.

$22 \%$ der anfänglich in der Deponie abgelagerten Kohlenstoffmenge werden im Deponiegas und nur $0.3 \%$ im Sickerwasser abtransportiert. $5 \%$ des Fluors werden durch das Deponiegas und circa $0.1 \%$ durch das Sickerwasser exportiert. Beim Chlor ist das Verhältnis umgekehrt: Etwa $6 \%$ des Chlors werden im Sickerwasser und weniger als $1 \%$ im Deponiegas exportiert. Weniger als $1 \%$ des Schwefels werden durch die beiden Produktströme exportiert.
Tabelle 5. Jährliche mittlere Konzentration der wichtigsten Elemente im Sickerwasser nach der intensiven Reaktorphase und Anforderungen an Einleitungen in ein Gewässer.

\begin{tabular}{lcc}
\hline Element & $\begin{array}{c}\text { Jährliche mittlere Konzentration } \\
\text { im Sickerwasser am Ende der } \\
\text { intensiven Reaktorphase } \\
\text { [mg/L] }\end{array}$ & $\begin{array}{c}\text { Anforderungen an Einleitungen } \\
\text { in ein Gewässer [23] }\end{array}$ \\
\hline $\mathrm{C}_{\text {org. }}$ & 750 & $10-15$ \\
$\mathrm{~F}^{\mathrm{m}}$ & 1200 & $5 \mathrm{~L}]$ \\
$\mathrm{P}$ & 0.7 & 10 \\
$\mathrm{~S}$ & 7 & 0.8 \\
$\mathrm{Cl}$ & 3 & $30 \mathrm{~b})$ \\
$\mathrm{Cu}$ & 1300 & $100 \mathrm{c})$ \\
$\mathrm{Zn}$ & 0.1 & 0.5 \\
$\mathrm{~Pb}$ & 0.6 & 2 \\
$\mathrm{Cd}$ & 0.07 & 0.5 \\
$\mathrm{Hg}$ & 0.002 & 0.1 \\
\hline
\end{tabular}

a) Qualitätsziel (VO 1975). b) Zehnfaches des Qualitätsziels für die Summe $\mathrm{NH}_{3}+\mathrm{NH}_{4}{ }^{+}$ c) Qualitätsziel für Sulfat-Schwefel.

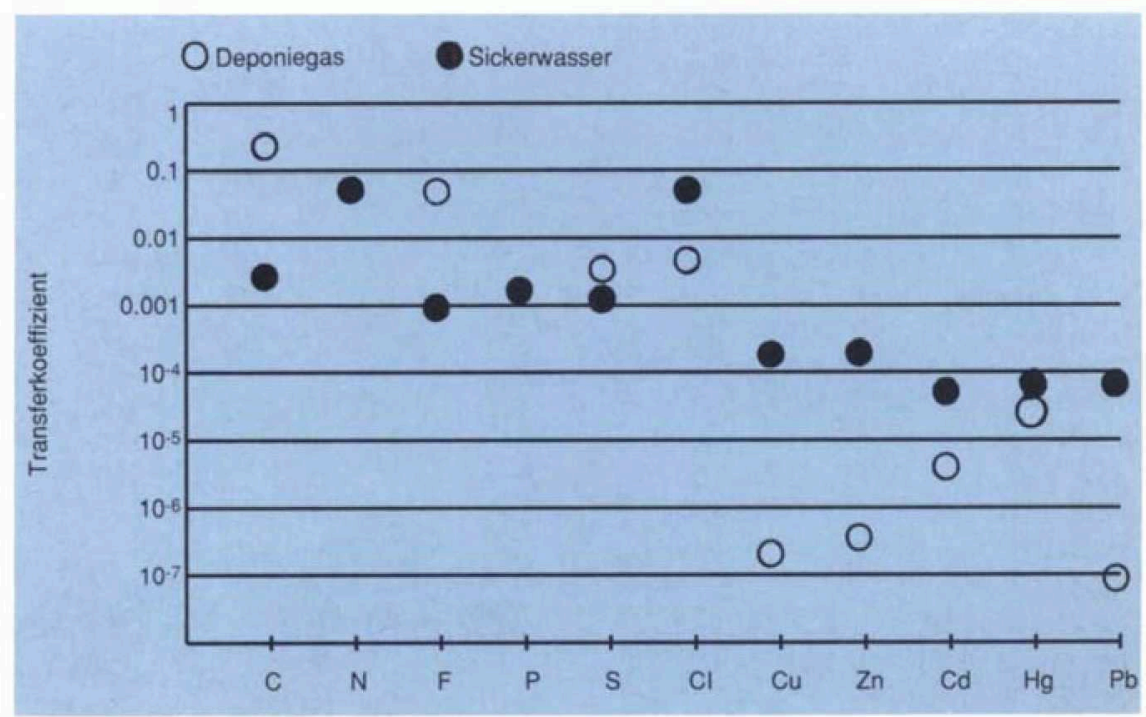

Figur 3. Transferkoeffizienten einiger Elemente am Ende der intensiven Reaktorphase. 
Die Metallkonzentrationen im Sickerwasser liegen unterhalb der Grenzwerte, ebenso Fluorid- und Schwefelkonzentrationen. Die Konzentrationen des gelösten organischen Kohlenstoffs (DOC), des Stickstoffs und des Chlorids sind jedoch bis zu zwei Größenordnungen höher als die Grenzwerte. Deshalb müssen die Sickerwässer behandelt werden, bevor man sie in ein Gewässer einleitet.

Wie lange müssen die Sickerwässer einer solchen Deponie behandelt werden? Um das Langzeitverhalten des Sickerwassers abzuschätzen, wurden Feldexprimente an bestehenden Deponien und Laboratoriumsexperimente durchgeführt 17.24.25!. Die Resultate wurden mit einem mathematischen Modell kombiniert, um die Elementfrachten im Sickerwasser als Funktion der Zeit nach der intensiven Reaktorphase zu prognostizieren. Die Zeit bis zum Erreichen der umweltverträglichen Frachten wurde dann abgeschätzt (vergleiche Figur 2 und 4). Fluor-, Schwefel- und Metallkonzentrationen erreichen die Grenzwerte während der intensiven Reaktorphase (mittlere Verweilzeit weniger als 10 Jahre). Stickstoffkonzentrationen oberhalb der Grenzwerte werden während mindestens mehreren Jahrzehnten erwartet. Die längsten Zeitspannen benötigen Chlor, Phosphor und der organische Kohlenstoff, nämlich mindestens ein bis mehrere Jahrhunderte.

Diese Resultate zeigen, daß die heutigen Reaktordeponien für Siedlungsabfälle zu Altlasten von morgen werden, da die Sickerwässer über mehrere Menschengenerationen behandelt werden müssen. Unter "Altlast" versteht man in der Entsorgung eine vom Menschen geschaffene Ablagerung, die wegen ihrer tatsächlichen oder möglichen Emissionen zum Schutze von Mensch und Umwelt behandelt oder saniert werden muß. Organische Stoffe sind entscheidend für die Dauer der Behandlung oder Sanierung. Unsere Kenntnisse der organischen Stoffe, die von den Reaktordeponien emittiert werden, sind noch sehr lückenhaft. Tausende von xenobiotischen Substanzen treten im Siedlungsabfall auf. Einige von ihnen können in den Deponien leicht abgebaut werden, andere lassen sich sehr schwer oder auch in Jahrhunderten nicht verändern. Eine andere Gruppe sind Substanzen, die in toxische Komponenten umgewandelt werden, selbst aber nicht toxisch sind. $\mathrm{Da}$ sehr wenig über die chemische $\mathrm{Zu}$ sammensetzung, die Ökotoxikologie und das Langzeitverhalten aller dieser Stoffe bekannt ist, müssen die Grenzwerte für den Summenparameter des gesamten

in Lösung befindlichen organischen Kohlenstoffs (DOC) wenigstens auf dem heute gewählten Niveau bleiben. Sie dürfen nicht hinaufgesetzt werden in der Absicht, die erforderlichen Behandlungszeiten $\mathrm{zu}$ verkürzen. In einer Gesamtbetrachtung kann postuliert werden, daß Reaktordeponien aus den in Tabelle 2 aufgeführten Abfällen mittel- und langfristig die lokalen und regionalen Wasserressourcen gefährden, hauptsächlich durch große Vielfalt organischer Verbindungen.

\subsection{Abschätzung der Immissionen aus Deponiealtlasten}

Zur groben Schätzung der aus Deponiealtlasten zu erwartenden Immissionen wurde der "Metaland"-Rahmen gewählt (Tabelle 1). Das Deponieinventar von 40 Tonnen pro Einwohner ( $t / E)$ ergibt

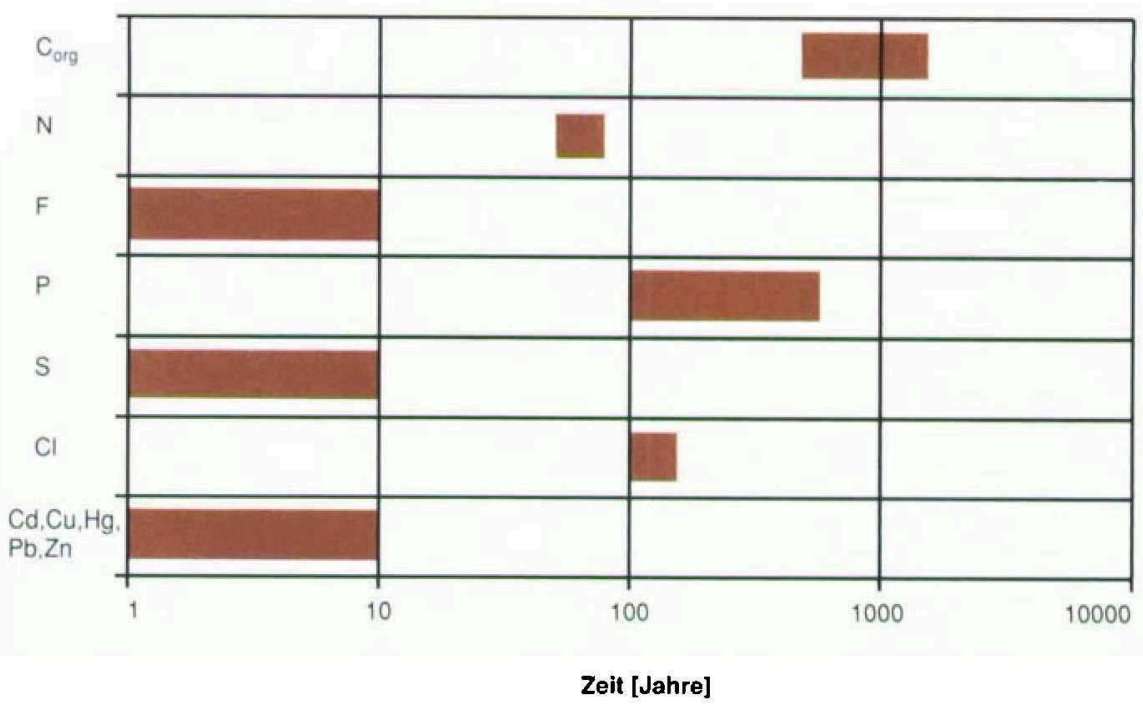

Figur 4. Abgeschätzte Zeitspannen, bis umweltverträgliche Frachten im Sickerwasser einer Siedlungsabfalldeponie erreicht werden.

sich aus der in der in Tabelle 2 angegebenen Menge deponierten Materials von circa 0.8 Tonnen pro Einwohner und $\mathrm{Jahr}(\mathrm{t} / \mathrm{E} \cdot \mathrm{a})$ des gesamten Abfalls für die achtziger Jahre, hochgerechnet auf die vergangenen 40 Jahre, unter Berücksichtigung der Tatsache, daß in den früheren Jahrzehnten zwar weniger Abfall pro Einwohner erzeugt wurde, dafür aber größere Anteile deponiert wurden [14] (siehe auch Figur 1). Zudem wird aufgrund der stofflichen Zusammensetzung des gesamten Abfalls (Tabelle 3) angenommen, daß sich dieser in erster Näherung ähnlich verhält wie ein Siedlungsabfall (Tabelle 5). Die Anzahl der Deponien beläuft sich auf etwa 1 pro 1000 Einwohner, das heißt, die mittleren Ausmaße betragen ungefähr 0.5 Hektar
Fläche und 10 Meter Tiefe bei 1.1 Tonnen pro Kubikmeter Dichte.

Tabelle 6 zeigt eine grobe Schätzung für die jährliche Konzentrationszunahme im Grundwasser von "Metaland" für fünf Indikatorelemente, wenn die Grundabdichtungen der Siedlungsabfalldeponien nicht mehrfunktionstüchtig sind und das Sickerwasser ins Grundwasser infiltriert. Es wurde angenommen, daß die Abdichtungen nach 50 Jahren durchlässig werden ${ }^{10}$ und das gesamte Sickerwasser ins Grundwasser gelangt. Biologische Abbauprozesse oder Adsorption im Aquifer wurden nicht berücksichtigt. Nach 50 Jahren ist die Konzentrationsabnahme von Zink, Cadmium und Quecksilber im Sickerwasser nicht signifikant. Voraussichtlich nehmen die Konzentrationen von organischem Kohlenstoff und Chlor in der gleichen Zeitspanne stärker ab. Bezüglich dieser Elemente ist jedoch das Sickerwasser um etwa

Zeit [Jahre]

zwei Größenordnungen konzentrierter als das nicht kontaminierte Grundwasser Deshalb sind durch das infiltrierte Sickerwasser hohe lokale Kontaminationen des Grundwassers in der nächsten Umgebung der Deponie zu erwarten. Eine regionale Erhöhung der Grundwasserkonzentration ist aber nur für den organischen Kohlenstoff signifikant. Besonders xenobiotische Substanzen mit hoher Mobilität und geringerer Abbaubarkeit können die Grundwasserqualität wesentlich verschlechtern. Mit anderen Worten: Die bereits bestehenden Reaktordeponien, welche noch heute zum Teil mit Emissionskontrolle geführt werden, bilden für die weitere Zukunft ein hohes Belastungspotential für das regionale Grundwasser. 
Tabelle 6. Abschätzung der jährlichen Konzentrationszunahme im Grundwasser, nachdem die Abdichtungen der Siedlungsabfalldeponien nicht mehr funktionstüchtig sind. Siedlungsabfallmenge in der Deponie: 40000 Kilogramm pro Einwohner; spezifischer Sickerwasserflux a): 0.02 Liter pro Kilogramm Siedlungsabfall und Jahr; Grundwasserreservoir: $2.0 \times 10^{9}$ Kubikmeter.

\begin{tabular}{|c|c|c|c|c|c|}
\hline & $\mathrm{C}_{\text {org. }}$ & $\mathrm{Cl}$ & $\mathrm{Zn}$ & $\mathrm{Cd}$ & $\mathrm{Hg}$ \\
\hline $\begin{array}{l}\text { Mittlere Konzentration } \\
\text { im Sickerwasser am } \\
\text { Ende der intensiven } \\
\text { Reaktorphase }[7] \\
\text { [mg L-1] }\end{array}$ & 750 & 1300 & 0.6 & 0.002 & 0.0005 \\
\hline $\begin{array}{l}\text { Mittlere Konzentration } \\
\text { im Sickerwasser nach } \\
50 \text { Jahren } \\
\text { [mg L-1] }\end{array}$ & 600 & 500 & 0.6 & 0.002 & 0.0004 \\
\hline $\begin{array}{l}\text { Mittlere Konzentration } \\
\text { im nicht kontaminienen } \\
\text { Grundwasser [37] } \\
\text { [mg L-1] }\end{array}$ & 0.5 & 3 & 0.005 & 0.00002 & 0.000005 \\
\hline $\begin{array}{l}\text { Mittlere spezifische } \\
\text { Elementfracht } \\
{\left[g E^{-1} a^{-1}\right]}\end{array}$ & 480 & 400 & 0.48 & 0.0016 & 0.0032 \\
\hline $\begin{array}{l}\text { Totale Elementfracht } \\
{\left[\mathrm{kg} \mathrm{a}^{-1}\right]}\end{array}$ & 480000 & 400000 & 480 & 1.6 & 0.32 \\
\hline $\begin{array}{l}\text { Mittlere jährliche } \\
\text { Konzentrations- } \\
\text { zunahme a) } \\
{\left[m g ~ L^{-1} a^{-1}\right]}\end{array}$ & 0.24 & 0.2 & 0.00024 & $8 \times 10^{-7}$ & $1.6 \times 10^{-7}$ \\
\hline $\begin{array}{l}\text { Mittlere jährliche } \\
\text { Konzentrations- } \\
\text { zunahme a) } \\
{[\%]}\end{array}$ & 50 & 7 & 5 & 4 & 3 \\
\hline
\end{tabular}

a) Berechnet für das gesamte Grundwassenorkommen.

\subsection{Behandlungstechniken für Altlasten}

Aus Abschnitt 3.3 geht hervor, daß die bestehenden Reaktordeponien zur Sicherung der Grundwasserqualität verfahrens- und bautechnisch geführt werden müssen. Es gibt verschiedene Methoden zur Verminderung der Umweltgefährdung durch solche Altlasten. Sie können in zwei Gruppen eingeteilt werden ${ }^{26 \mid}$ :

\section{Techniken zur Sicherung}

Wenn die Abdichtungen einer Deponie nicht mehr funktionstüchtig sind, infiltriert das Sickerwasser in das Grundwasser. Eine Kontamination des Grundwassers durch Schadstoffe muß dann mit nachträglich hergestellten Abdichtungen verhindert werden. Dazu gehören Oberflächenabdichtungen zur Reduktion der Wasserzufuhr sowie vertikale und horizontale Dichtungswände. Diese Techniken werden meistens in Kombination mit hydraulischen Maßnahmen angewendet. Durch Absenken des Grundwasserspiegels wird die Ausbreitung des schon kontaminierten Grundwassers verhindert. Das gesammelte Sickerwasser muß jedoch weiterhin behandelt werden. Da alle diese Methoden das Schadstoffpotential der Altlasten nicht vermindern oder be- seitigen können, werden sie solange benutzt, bis langfristige Lösungen für das Problem gefunden werden.

\section{Techniken zur Sanierung}

Eine Verminderung oder Beseitigung des Schadstoffpotentials der Altlasten wird durch Verfahrenstechniken zur Sanierung möglich gemacht. Dafür stehen thermische, chemisch-physikalische und biologische Verfahren zur Verfügung. Thermische Verfahren werden für die Behandlung hochkonzentrierter organischer Schadstoffe verwendet. Das Prinzip ist immer eine Oxidation bei erhöhten Temperaturen $\left(600\right.$ bis $1300^{\circ} \mathrm{C}$ ). Die Anlagen bestehen meistens aus Drehrohröfen oder Wirbelschichtöfen. Als chemisch-physikalische Verfahren kommen Waschprozesse in Frage. Hierbei werden Schadstoffe mit einem Extraktionsmittel ausgewaschen. Sowohl bei den thermischen als auch bei den chemisch-physikalischen Verfahren werden deponierte Materialien ausgegraben und vor Ort oder nach dem Abtransport in spezialisierten Anlagen behandelt. Biologische Verfahren können sowohl nach der Ausgrabung des Materials als auch in natürlicher Lage angewendet werden, um organische Verbindungen durch Stoffwechselprozesse abzubauen. Eine weitere Variante, die in situ angewendet wird, ist ein hydraulisches Verfahren: Das Grundwasser wird durch Entnahmebrunnen gefördert, vor Ort gereinigt und wieder gezielt durch die Altlast versickert. Auf diese Weise werden Schadstoffe ausgewaschen. Ein solches Verfahren kann auch mit biologischen Verfahren kombiniert werden, indem zum Beispiel dem gereinigten Grundwasser vor der Zurückführung Mikroorganismen zugesetzt werden.

In der relativ kurzen Entwicklungsphase vom Erkennen der ersten größeren Altlasten bis heute wurden zahlreiche Sicherungs- und Sanierungstechniken entwickelt. Alle verlangen einen erheblichen finanziellen Aufwand. So ist in der Schweiz mit einem Aufwand pro zu sanierende Deponie von 0.5 bis 50 Millionen Franken zu rechnen, das entspricht 10 bis 1000 Franken pro eingelagerte Tonne, je nach gewähltem Verfahren. Für die ganze Schweiz würde dies nach heutigen Preisen (1991) einen Gesamtaufwand von einigen Milliarden Franken bedeuten, allerdings verteilt über mehrere Jahre bis Jahrzehnte. Gemessen an den gesamten jährlichen Entsorgungsausgaben von zur Zeit mehreren Milliarden pro Jahr erscheint dies als keine untragbare zusätzliche Belastung. Das Hauptproblem liegt vielmehr auf der psychologisch-politischen Ebene ${ }^{121}$. Die öffentliche Hand wird in den meisten Füllen gezwungen sein, Steuergelder für diese Aufgaben einzusetzen, da ein Ruickgriff auf die Verursacher nicht mehr möglich ist. Darüber muß die Öffentlichkeit schleunig aufgeklärt werden, um das notwendige Verständnis für zusätzliche Kredite zu schaffen. Denn es gibt keine Alternative zur Bewältigung dieser stofflichen Vergangenheit.

Weltweit arbeiten schon viele Forschungsgruppen an der Evaluierung und Sanierung von Altlasten. Das Schwergewicht liegt dabei auf den verfahrens- und bautechnischen Verfahren. In praktisch allen Sanierungsfällen fehlen genügende Kenntnisse über die chemische Zusammensetzung des Deponieinhalts, da keine genügende Dokumentation während des Deponieaufbaus angelegt wurde. Es ist sehr schwierig und aufwendig, nachträglich aus Bohrungen die Zusammensetzung solcher heterogenen Ablagerungen mit hinreichender Genauigkeit zu bestimmen. Große Lücken klaffen auch im Verständnis der deponieinternen Prozesse. Die Steuerung des Wasserhaushaltes einerseits sowie der mikrobiellen Reaktionen anderseits bedürfen somit viel grundlagenorientierter Forschung. 


\section{Entwicklungsszenarios für anthropogene Sedimente}

\subsection{Die geogenen Sedimentationsprozesse als Modell}

Im Grenzbereich der Erdkruste zu Atmo-, Hydro- und Biosphäre entstehen natürlicherweise seit Jahrmilliarden Ablagerungen. Die geogene Sedimentation, verstanden als vom Menschen unbeeinflußte Ablagerungs- und Akkumulationsprozesse an der Erdoberfläche, geschieht im wesentlichen durch

- Erosion und Wiederablagerung der Erosionsprodukte, bedingt durch die örtliche Topographie und die Gravitationskräfte;

- Ablagerung von Organismenresten am Boden aquatischer, seltener terrestrischer Ökosysteme;

- Ablagerung vulkanischer Emissionen; - chemische Ausfällung in Gewässern.

Die beiden erstgenannten Mechanismen sind die quantitativ bedeutendsten. Bei jeder Ablagerung setzen Prozesse ein, die Veränderungen im Sedimentkörper bewirken. Unverfestigte Sedimente zersetzen sich oder wandeln sich über Jahrtausende zu Sedimentgesteinen um. Auch verfestigte Ablagerungen können sich in ihren Materialeigenschaften verändern. Die Gesamtheit der Transformationsprozesse in Sedimenten und Gesteinen wird als Diagenese bezeichnet, wenn das Substrat nicht direkt den atmosphärischen Bedingungen ausgesetzt ist (Figur 5, Szenario 1 und 3).

Findet ohne Überlagerung ein direkter Kontakt zur Atmosphäre statt, so setzt Verwitterung ein (Figur 5, Szenario 2). Die Verwitterung bewirkt eine physikalische Zersetzung (mechanische Verwitterung) und eine chemische Veränderung (chemische Verwitterung oder Alteration). Sie ist gekoppelt mit der Erosion. Sofern die Erosion langsamer ist als die chemische Verwitterung, kommt es zur Bodenbildung. Verwitterung und Diagenese sind konkurrierende Prozesse. Erfolgt die Erosion des abgelagerten Materials schneller als der Nachschub neuer Sedimente (Figur 5, Szenario 2), so kommt es - zumindest unter nicht aquatischen Bedingungen - zur Verwitterung. Insbesondere bei terrestrischen Ablagerungen, zum Beispiel bei Windablagerungen (Löß) oder bei anthropogenen Ablagerungen (Deponien) können diagenetische Prozesse und Verwitterungsprozesse aber auch gleichzeitig ablaufen: Während in tieferen Bereichen diagenetische Prozesse überwiegen, findet nahe der Sedimentoberfläche
Verwitterung statt. Bleibt die Ablagerung unbedeckt, so wird sich die Verwitterungszone mit fortschreitender Erosion nach unten verlagern. Die Geschwindigkeit der Verlagerung wird also durch die Erosionsrate bestimmt. Die Erosi-
Szenario 1
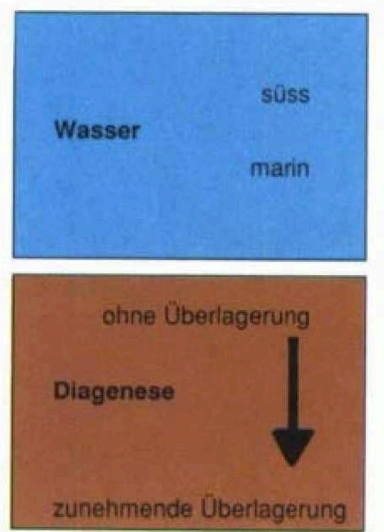

Szenario 2
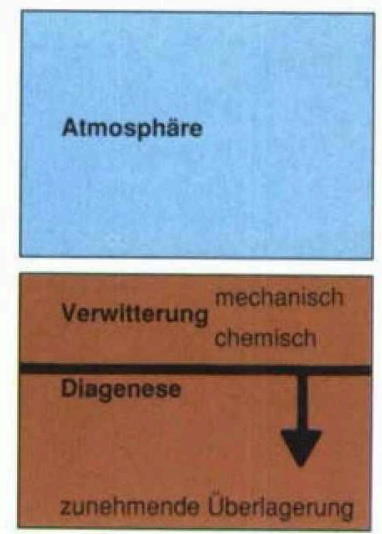

Sediment
Sedimente und Sedimentgesteine lassen sich mit Einschränkungen auch auf anthropogene Sedimente übertragen. Es kann von der Hypothese ausgegangen werden, daß selbst in Ablagerungen mit naturfremden Substanzen die meisten

Szenario 3
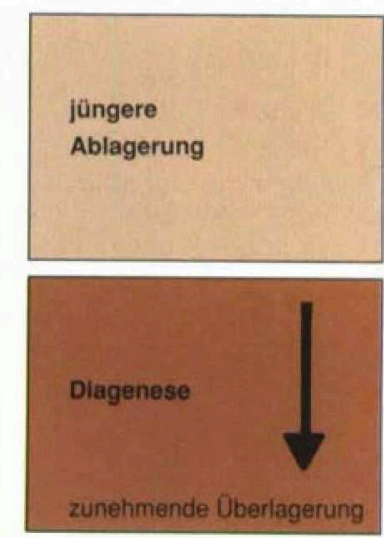

Figur 5. Lagerungsbedingungen für Sedimente. Szenario 1: Die Sedimente sind mit Wasser überdeckt; Szenario 2: Die obersten Sedimente sind im Kontakt zur Atmosphäre; Szenario 3: Die Sedimente werden von jüngeren überlagert. onsraten unserer Modellregion betragen $5-100 \mathrm{~cm}$ pro 1000 Jahre (Werte der Schweiz). Für die vollständige Verwitterung einer $25 \mathrm{~m}$ mächtigen Ablagerung wären demnach zumindest 25000 Jahre erforderlich (Figur 6).

Die Effizienz der Verwitterung wird aber nicht allein durch die Größe der erosiven Kräfte bestimmt, sondern ist auch eine Funktion der Feststoffeigenschaften des Sediment- oder Gesteinskörpers. Die Feststoffeigenschaften sind auch für den Diageneseverlauf von entscheidender Bedeutung. Sowohl im Verwitterungsszenario als auch im urteilung der langfristigen Umweltverträglichkeit anthropogener Sedimente eine Entwicklung abgeschätzt werden, die sich über Jahrtausende erstreckt. Erkenntmisse aus dem Studium geogener Szenario der Diagenese muß bei der Be-
Prozesse prinzipiell gleich sind wie in natürlichen Ablagerungen. Auch wenn sich diese Hypothese erst nach geologischer Erfahrungszeit vollständig validieren lassen wird, so ist sie sicherlich insoweit richtig und im Sinne des Vorsorgeprinzips, daß die "Experimente", die in der Natur über Jahrmilliarden ablaufen, für die Beurteilung anthropogener Sedimente genutzt werden können. Zumindest können am Beispiel geooder biogener Sedimente und Gesteine die Eigenschaften abgeleitet werden, die für das Verhalten von Ablagerungen generell relevant sind. Für die in $A b$ schnitt 3 beschriebenen Reaktordeponien als eine Form anthropogener Sedimentation gilt vorerst die Feststellung, daß die ersten Verlagerungs-, Akkumulations- und Ablagerungsprozesse anthropogener Natur sind, das heißt, es

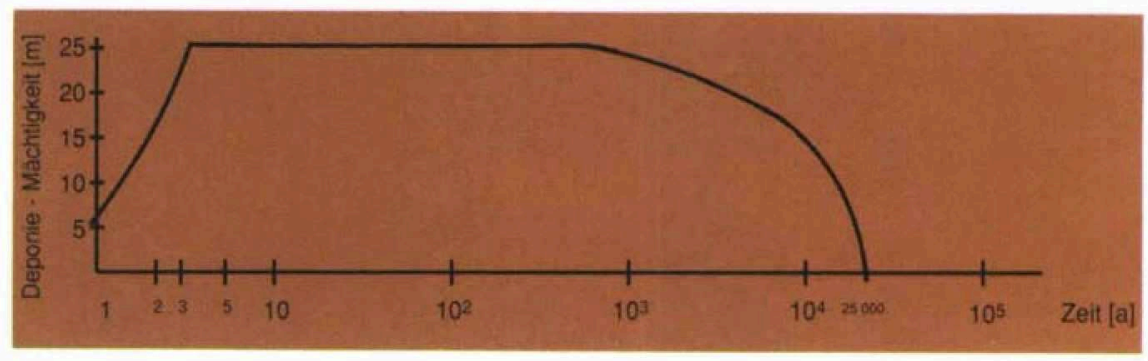

Figur 6. Auffüllung und Erosion einer 25 Meter mächtigen Deponie, dargestellt auf einer logarithmischen Zeitskala. Bei einer sehr hohen Erosionsrate von 100 Zentimeter pro 1000 Jahre sind für die vollständige Erosion 25000 Jahre erforderlich. 
werden auf Fahrzeugen Materialien eingesammelt und in geo- und bautechnisch vorbereiteten Geländezonen akkumuliert und eingebaut. Erst dann erfolgen, parallel zu anthropogen geführten Prozessen wie etwa der Sickerwasserfassung, die bereits erwähnten geogenen Prozesse, wobei $\mathrm{zu}$ berücksichtigen ist, $\mathrm{da} \beta$ durch die vergleichsweise sehr schnelle Sedimentationsrate große Mächtigkeiten mit gleichem diagenetischem Potential geologisch gesehen fast gleichzeitig zur Ablagerung kommen. Will man die in Abschnitt 2 begründeten Ziele für anthropogene Sedimente erreichen, so ist es unerläßlich, ihre frühe Diagenese zu verstehen und die Qualitätsanforderungen für ihre Produkte zu definieren. Kurzfristig (Jahre bis Jahrzehnte) betrifft dies vor allem die Emissionen in Luft und Wasser, mittel- und langfristig (Jahrzehnte bis Jahrhunderte) den festen Rückstand und seine geochemischen Eigenschaften.

\subsection{Die geochemischen Eigenschaften der Erdkruste als Modell}

Ein Vergleich der chemischen Zusammensetzung anthropogener Stoffgemische mit der Zusammensetzung der Erdkruste kann in erster Näherung als $\mathrm{MaB}$ für deren Ressourcen und Emissionspotential herangezogen werden. Die Erdkruste besteht zu $95 \%$ aus magmatischen Gesteinen; die sedimentären Gesteine nehmen nur $5 \%$ des Volumens ein ${ }^{|27|}$. Die Kruste ist $30 \mathrm{~km}$ mächtig (kontinentale Kruste), respektive $5 \mathrm{~km}$ (ozeanische Kruste). Die an der Erdoberfläche aufgeschlossenen Gesteine sind zu $75 \%$ aus Sedimenten entstanden (Figur 7). Anthropogene Ablagerungen finden in den äußersten Krustenbereichen statt. Sie sollten daher nicht alleine mit den Durchschnittswerten der gesamten Kruste verglichen werden, sondern auch mit den in Sedimentgesteinen üblichen Variationsbreiten (Tabellen 7 und 8).

Geogene Festkörper, die bezüglich eines oder mehrerer Elemente im Vergleich zum geochemischen Durchschnitt stark angereichert sind, werden als Erze bezeichnet. Sie können durch magmatische Differentiation, hydrothermal, sedimentär oder als Folge von Metamorphose und Verwitterung entstehen. Erze haben nicht nur ein für den Menschen höheres Wertstoffpotential als sonstige Gesteine, sondern zugleich auch ein höheres Schadstoffpotential. Inwieweit sie die Umwelt beeinflussen, ist abhängig von den Feststoffeigen-

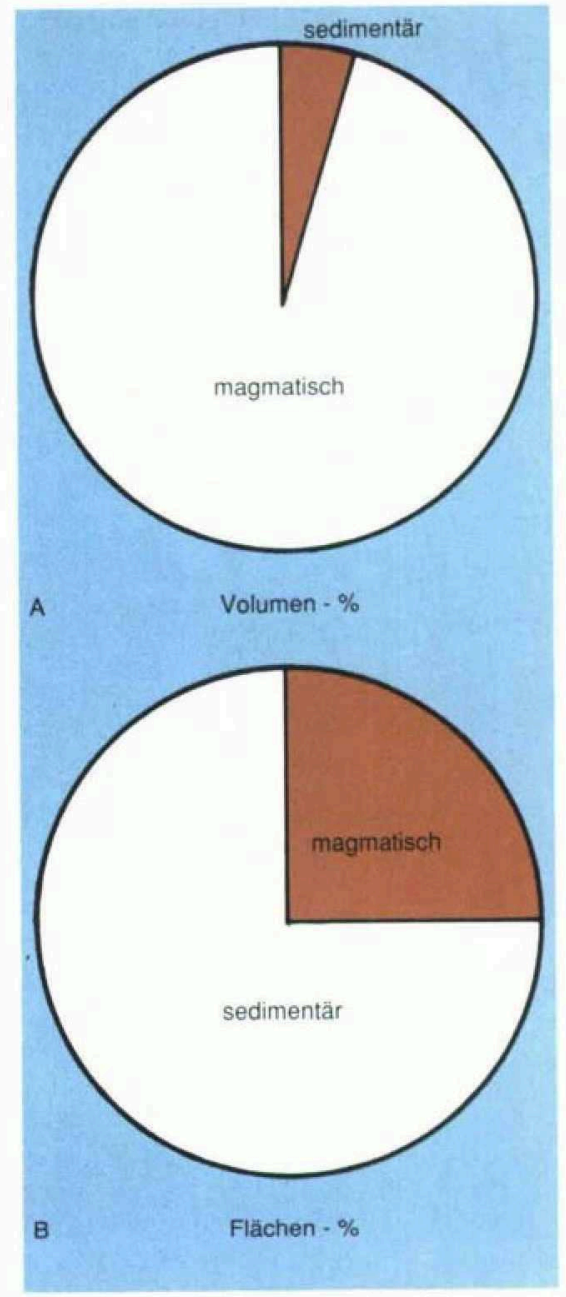

Figur 7. Relative Häufigkeit magmatischer und sedimentärer Gesteine in der Erdkruste [27], A: bezogen auf das Krustenvolumen, $B$ : bezogen auf die äußerste Kruste (Oberfläche) schaften, aber auch von den Lagerungsbedingungen. So gibt es eine an Erzlagerstätten angepaßte Vegetation. In Lagerstätten, die der Verwitterung ausgesetzt waren, konnte sich eine auf hohe Schwermetallkonzentrationen spezialisierte Flora entwickeln ${ }^{[28]}$. Aus solchen Gebieten stammende Gewässer weisen stark erhöhte Schwermetallkonzentrationen auf, welche auf manche Organismen toxisch wirken. Die in Abschnitt 2 formulierte Zielsetzung der "Erdkrustenähnlichkeit" für anthropogene Sedimente ist demnach differenziert anzusteben, das heißt nach Maßgabe ihres geochemischen Umfelds.

Die Abfallbewirtschaftung sollte so optimiert werden, daß die großen Mengen von nicht verwertbaren Feststoffen möglichst gesteinsähnlich anfallen, also von potentiellen Schadstoffen entlastet werden. Nicht direkt wiederverwertbare Spurenstoffe (zum Beispiel Schwermetalle aus Elektrofilterstäuben; Tabelle 8) sollten konzentriert und unter entsprechenden Bedingungen als anthropogene Erze gelagert werden. Diese Optimierung kann einerseits durch Vorsortierung oder die Vermeidung von Vermischung, anderseits durch Behandlungsverfahren (mechanisch, thermisch, chemisch, biologisch) erreicht werden.

\subsection{Die Deponietypen der Zukunft}

Entsprechend der Unterscheidung in Gesteine und Erze werden für die Endlagerung (vergleiche Abschnitt 2) also zwei Deponietypen benötigt:
Tabelle 7. Vergleich von Schlacke und Elektrofilterstaub aus Schweizer Kehrichtverbrennungsanlagen mit Gesteinen und Erzen bezüglich der Matrixelemente.

$\begin{array}{ccc}\begin{array}{c}\text { Verbrennungs- } \\ \text { rückstände }[15] \\ {[g / k g]}\end{array} & \text { Gesteine (Erdkruste) }{ }^{[29]} & \begin{array}{c}\text { Erze } \\ \text { (Rohstoffgehalte) [30] } \\ {[\mathrm{g} / \mathrm{kg}]}\end{array}\end{array}$

Elemente Schlacke E-Filterstaub Variationsbreite a) Mittelwert ${ }^{\text {b) }}$ (Matrix)

\begin{tabular}{|c|c|c|c|c|c|c|}
\hline 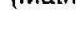 & & & & odell $A$ & Modell $B$ & \\
\hline $\mathrm{SiO}_{2}$ & $360-460$ & $230-320$ & $51-744$ & 666 & 583 & n. b. \\
\hline $\mathrm{Ca}^{2}$ & $25-100$ & $30-90$ & $5-302$ & 26 & 51 & n. b. \\
\hline $\mathrm{Fe}$ & $40-230$ & $28-40$ & $3.8-94$ & 34 & 58 & $400-600$ \\
\hline$C_{\text {total }}$ & $15-40$ & $14-36$ & n. b. & n. b. & 0.2 & n. b. \\
\hline $\mathrm{C}^{\text {lotal }}$ & $10-20$ & $10-30$ & $0-10^{c)}$ & 0 & 0 & n. b. \\
\hline $\mathrm{Mg}$ & $6-18$ & $28-40$ & $1.6-204$ & 33 & 28 & $10-30$ \\
\hline Al & $80-180$ & $60-120$ & $4.2-88$ & 77 & 80 & $250-350$ \\
\hline$K$ & $5-20$ & $12-74$ & $0.03-48$ & 30 & 17 & n. b. \\
\hline $\mathrm{Na}$ & $10-60$ & $20-80$ & $0.2-40$ & 32 & 23 & 400 \\
\hline$S$ & $2-4$ & $20-40$ & $0.2-2.4$ & 0.3 & 0.3 & n. b. \\
\hline & Sulfat & Sulfat & & & & \\
\hline$N$ & n. b. & n. b. & $0.006-$ & 0.02 & 0.02 & n. b. \\
\hline $\mathrm{Cl}$ & $3-6$ & $40-78$ & $0.02-0.43$ & 0.21 & 0.19 & 600 \\
\hline$P$ & $7-14$ & $1-12$ & $0.17-1.1$ & 0.82 & 1.0 & 200 \\
\hline
\end{tabular}

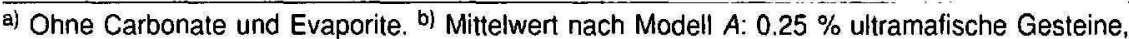
$18 \%$ Basalt, $78 \%$ Granit, $3.75 \%$ Syenit; Mittelwert nach Modell B: $50 \%$ Basalt, $50 \%$ Granit. c) $C_{\text {org }}: 10=$ Mittelwert in Sedimentgesteinen. (n. $b .=$ nicht bestimmt). 
Tabelle 8. Vergleich von Schlacke und Elektofilterstaub aus Schweizer Kehrichtverbrennungsanlagen mit Gesteinen und Erzen bezüglich der Spurenelemente.

$\begin{aligned} & \text { Verbrennungs- } \\ & \text { rückstände [15] } \\ & {[\mathrm{mg} / \mathrm{kg}]}\end{aligned}$
$[\mathrm{mg} / \mathrm{kg}]$

Erze
$\begin{gathered}\text { (Rohstoffgehalte) } \\ {[\text { [30] }}\end{gathered}$
$[\mathrm{mg} / \mathrm{kg}]$

Elemente Schlacke E-Filterstaub Variationsbreite Mittelwert ${ }^{\text {a) }}$ (Spuren)

\begin{tabular}{|c|c|c|c|c|c|c|}
\hline & & & & odell $A$ & Modell $E$ & \\
\hline $\mathrm{Zn}$ & $\begin{array}{l}4000- \\
15000\end{array}$ & $\begin{array}{l}13000- \\
39000\end{array}$ & $16-130$ & 63 & 82 & $\begin{array}{l}40000 \\
50000\end{array}$ \\
\hline $\mathrm{Cu}$ & $\begin{array}{l}1000- \\
4000\end{array}$ & $\begin{array}{l}1000- \\
5000\end{array}$ & $4-87$ & 32 & 58 & $\begin{array}{l}5000- \\
20000\end{array}$ \\
\hline $\mathrm{Pb}$ & $\begin{array}{l}1000- \\
17000\end{array}$ & $\begin{array}{l}6000- \\
12000\end{array}$ & $1-20$ & 15 & 10 & $\begin{array}{l}20000- \\
50000\end{array}$ \\
\hline $\mathrm{Ba}$ & n. b. & n. b. & $0.4-1600$ & 610 & 380 & n. b. \\
\hline $\mathrm{Mn}$ & n. $b$. & n. b. & 0.1500 & 670 & 1000 & 200000 \\
\hline $\mathbf{F}$ & n. b. & n. b. & $100-1200$ & 650 & 460 & n. b. \\
\hline $\mathrm{Cr}$ & $\begin{array}{l}1000- \\
10000\end{array}$ & $\begin{array}{l}500- \\
1700\end{array}$ & $4-2980$ & 48 & 96 & 300000 \\
\hline $\mathrm{Ni}$ & 300 & $200-300$ & $2-2000$ & 37 & 72 & $\begin{array}{l}10000- \\
20000\end{array}$ \\
\hline B & n. b. & n. b. & $3-100$ & 9 & 7 & n.b. \\
\hline $\mathrm{Sn}$ & n. b. & n. b. & $0-6$ & 2.1 & 1.5 & $\begin{array}{c}200- \\
10000\end{array}$ \\
\hline As & n. b. & n. b. & $1-13$ & 1.7 & 2.0 & n. b. \\
\hline $\mathrm{Cd}$ & 30 & $200-600$ & $0.03-0.3$ & 0.15 & 0.18 & $\begin{array}{l}5000- \\
30000\end{array}$ \\
\hline $\mathrm{Hg}$ & 0.1 & $2-14$ & $0.004-0.4$ & 0.003 & 0.02 & $(\text { (n. b. })^{\text {b) }}$ \\
\hline
\end{tabular}

a) Mittelwert nach Modell A: $0.25 \%$ ultramafische Gesteine, $18 \%$ Basalt, $78 \%$ Granit, $3.75 \%$ Syenit; Mittelwert nach Modell $B: 50 \%$ Basalt, $50 \%$ Granit. b) In Zinnober ( $\mathrm{HgS}$ ): $\mathrm{Hg}=86.2 \%$. (n. b. = nicht bestimmt).

- Typ A : Deponie mit chemisch und physikalisch stabilen Stoffgemeinschaften mit geringem Wert- und Schadstoffpotential (gesteinsähnlich);

- Typ $B$ : Deponie mit chemisch und physikalisch stabilen Stoffgemeinschaften mit hohem Wert- und Schadstoffpotential (erzähnlich).

Stoffgemeinschaften, die noch nicht geniigend stabil sind, brauchen Betreuung und gehören somit nicht in ein Endlager. Für sie benötigt man einen dritten Deponietyp, die Reaktordeponie (siehe auch Abschnitt 3):

- Typ $C$ : Deponie, in der wegen der Stoffzusammensetzung chemische und/oder biologische Prozesse ablaufen, deren Produkte eine Nachbehandlung der Gase und/oder Sickerwässer notwendig machen.

Die Zuweisung zu einem Endlager setzt voraus, daß die Stabilität anthropogener Ablagerungen hinreichend beurteilt werden kann und genügend groß ist. Solange das nicht der Fall ist, sollten Deponien vorsorglich als $\mathrm{Re}$ aktordeponien eingerichtet werden. Unterschiedliche Stoffgemeinschaften dürfen aber auch dann nicht vermischt wer- den, sondern müssen getrennt in Monodeponien abgelagert werden. Die Trennung in gesteinsähnlich und erzähnlich gilt damit auch für die künftigen Reaktordeponien. Wir unterscheiden dann die Typen $C_{A}$ und $C_{B}$.

Definiert man Endlagerqualität als einen Zustand sehr geringer Reaktivität (geringes Potential chemischer Veränderungen), so sind grundsätzlich drei Szenarios denkbar, diesen zu erreichen (Figur 8). Als Maß für die Reaktivität kann die pro Zeit und pro Deponiemasse emittierte Stofffracht in die Umwelt gewählt werden.

1. In jeder Reaktordeponie gibt es Stoffe, welche die Forderung nach geringer Reaktivität von Anfang an erfüllen, und es gibt andere, die sie erst nach mehr oder weniger langer Lagerungszeit erfüllen. Durch eine Vorbehandlung kann diese Zeitspanne verkürzt werden.

2. Alle Stoffe werden vor ihrer Ablagerung soweit behandelt, daß sie die bis dahin bekannten Kriterien zur Beurteilung einer geringen Reaktivität erfüllen. Die Kriterien erweisen sich zur Zeit noch als ungenügend (nur Auslaugtests). Im Laufe der Diagenese und/oder Verwitterung verändern sich die Bedingungen derart, daß einzelne Stoffe mit Verzögerung zu reagieren beginnen (zeitlich verschobene Reaktorphase), so daß eine Beeinträchtigung der Umwelt nicht mehr ausgeschlossen werden kann.

3. Es gelingt, alle Stoffe vor ihrer Ablagerung in einen Zustand geringer Reaktivität umzuwandeln, und zwar derart, daß eine hohe Stabilität sowohl gegen Verwitterung als auch gegen Diagenese erreicht und beibehalten wird.

Solange durch die Vorbehandlung Prozesse in geraffter Zeit vorweggenommen werden, die nach der Ablagerung auch natürlicherweise zu erwarten wären, ist die Wahrscheinlichkeit für Szenario 2 sehr gering. Die Zeitspanne in Szenario 1 wird verkürzt, im Idealfall gemäß Szenario 3 auf Null reduziert. Schwieriger wird die Beurteilung von Verfahren, die sich nicht oder wenig an den in der Geosphäre zu erwartenden Prozessen orientieren. Hierzu wäre ein umfassenderes Verständnis der für das Langzeitverhalten relevanten Anforderungen die Voraussetzung.

Am Beispiel der für die Schweiz ab 1991 geltenden Technischen Verordnung Abfall ${ }^{1311}$ soll verdeutlicht werden, wie sich eine Umstellung auf die neuen Deponietypen vollziehen könnte. Neben gemischten Reaktordeponien (Typ $C$ ) werden neu "Inertstoffdeponien" (Typ $A$ ) und "Reststoffdeponien" (Typ $B$ ) eingeführt. Die Unterscheidung in $A$ und $B$ ist insofern identisch mit der vorher erörterten, als auch hier eine Trennung aufgrund des Schadstoffpotentials angestrebt wird. Monodeponien sind darüber hinaus zwar noch nicht zwingend vorgeschrieben; durch erleichterte Standortanforderungen im Falle der Monodeponierung wurde aber ein Anreiz geschaffen, solche Deponien zu betreiben. Die Zuweisung zu $A$ oder $B$ erfolgt uiber eine Beurteilung der chemischen Zusammensetzung (Summenparameter und Maximalwerte einiger Metalle für Typ $A$ ) und einen Eluattest, wobei für $A$ und $B$ unterschiedliche Grenzwerte einzuhalten sind. Bedingt durch den heute noch ungeniigenden Wissensstand einerseits und den Mangel an erprobten Behandlungsverfahren anderseits sind die stofflichen Vorschriften aber noch nicht so, daß diese Deponien als Endlager im Sinne der hier diskutierten Definition 


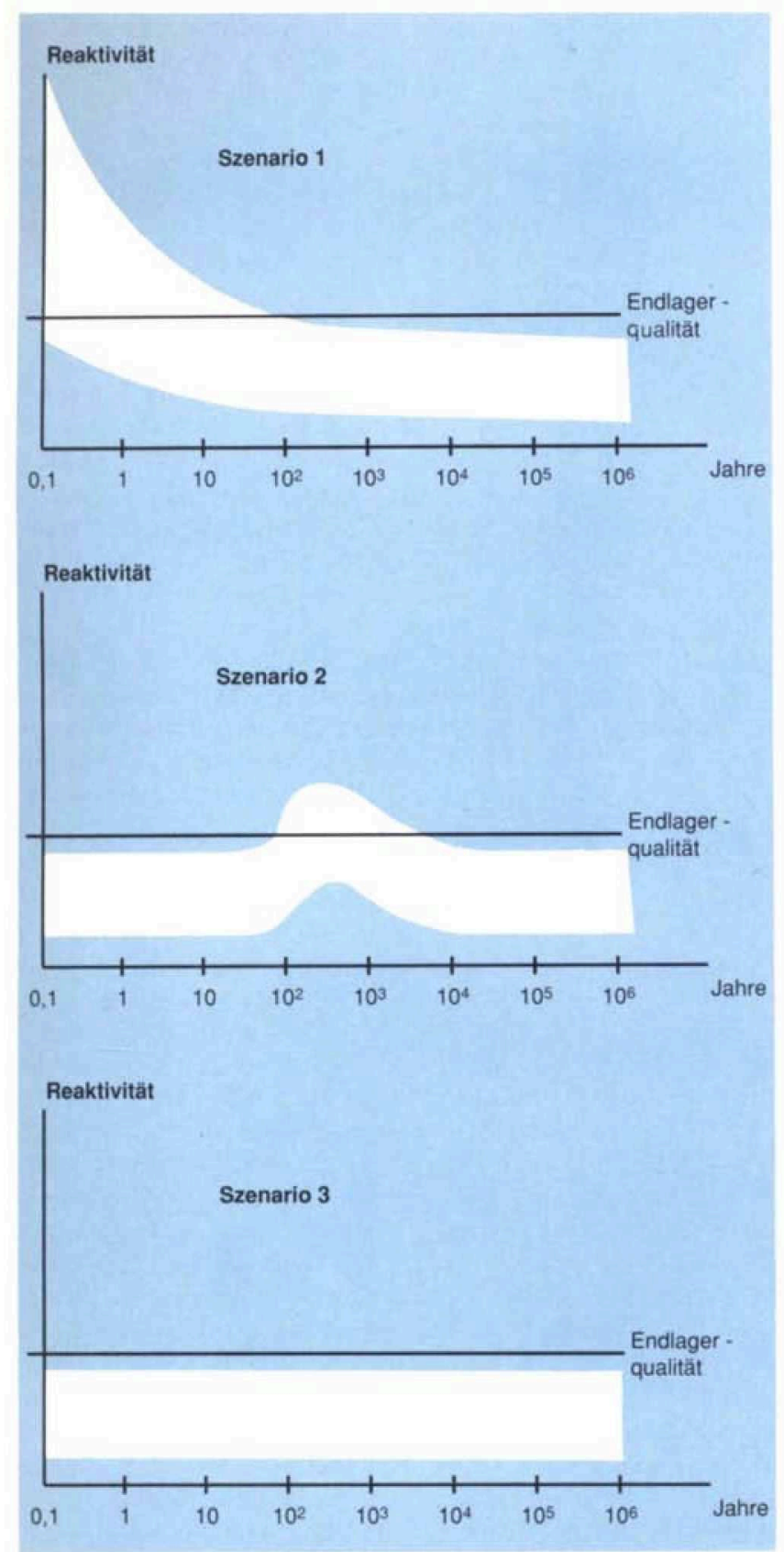

Figur 8. Die Reaktivität von Ablagerungen (Deponien) und ihre zeitliche Veränderung. Die Horizontale bei einer bestimmten sehr tiefen Reaktivität definiert die Endlagerqualität. Die drei Szenarios stellen unterschledliche stoffliche Entwicklungen dar. Szenario 3 entspricht dem Ideal der Endlagerqualität.

betrachtet werden könnten. Sie werden daher auch nicht als solche bezeichnet. Konsequenterweise muß man davon ausgehen, daß es sich auch bei diesen Ablagerungen um potentielle Reaktordeponien handelt; selbst dann, wenn sie zum Erreichen der Eluatgrenzwerte vorgängig behandelt wurden. In diesem Sinne sind die in der TVA verwendeten Begriffe "Inertstoffdeponien" als Typ $C_{A}$ und "Reststoffdeponien" als Typ $C_{B}$ zu verstehen. $D a$ sowohl bei $C_{A}$ als auch bei
$C_{B}$ ausschließlich Stoffgruppen mit relativ geringem organischem Anteil zur Ablagerung kommen, ist die Palette möglicher Reaktionen eingeschränkt, und es sind wesentlich kürzere Behandlungszeiten $\mathrm{zu}$ erwarten als bei herkömmlichen Reaktordeponien. Organische Abfälle, die nicht verwertbar sind (etwa zur Kompostherstellung), müssen also vor ihrer Ablagerung so behandelt werden, daß nur anorganische Rückstände entstehen. Thermische Verfahren, zum Beispiel Oxidation in einer Verbrennungsanlage (KVA; Pyrolyse mit nachgeschalteter Verbrennung, Sondermüllverbrennung), können und muissen nach heutigem Stand der Technik dafür verwendet werden. Verbrennungsanlagen lassen bereits in erster Näherung eine Auftrennung in Güter vom Typ $A$ und Typ $B$ zu, so daB eine derartige $\mathrm{Zu}-$ ordnung überhaupt erst möglich wird (Tabellen 7 und 8). Die in der TVA festgelegten Eluatanforderungen für Inert- respektive Reststoffdeponien lassen sich aber mit den heute in Betrieb stehenden Verbrennungsanlagen noch nicht erfüllen. Die Rückstände der Verbrennung müssen also weiter behandelt werden.

Diese erste Beurteilung der aktuellen Situation zeigt, daß bereits die Zielsetzung nach Deponiequalitäten der Typen $C_{A}$ und $C_{B}$

- kurzfristig (Jahre) eine wesentliche Modifizierung der heute gebräuch- lichen Behandlungsverfahren verlangt; - mittelfristig (Jahrzehnte) nur noch jene Versorgungsgüter zuläßt, welche dic Randbedingungen der so modifizierten Entsorgungssysteme erfüllen können; - schrittweise anthropogene Sedimente schafft, die im Hinblick auf ihre Qualität auch potentiellen Ressourcen für spätere Generationen nahekommen.

Alles in allem gibt es viele triftige Gründe dafür, die Entwicklung naturwissenschaftlich gestützter Beurteilungskriterien für anthropogene Sedimente mit Endlagerqualität als dringende Aufgabe zu sehen.

\subsection{Entwicklung naturwissenschaftlicher Kriterien zur Beurteilung der Endlagerqualität}

Das Verhalten von Ablagerungen wird durch deren Zusammensetzung und durch externe Faktoren bestimmt. Für das generelle Verhalten spielen die Hauptoder Matrixelemente eine weitaus wichtigere Rolle als die Spurenstoffe. Die Matrix bestimmt in Wechselwirkung mit externen Faktoren das chemische und physikalische Milieu in einer Ablagerung und damit auch das Verhalten von Spurenstoffen. Spurenstoffe können zwar ihrerseits Verschiebungen in der Entwicklung der Matrix bewirken; es wäre aber sicherlich ein falscher Ansatz, Beurteilungen der Umweltverträglichkeit allein auf "umweltrelevante" Spurenstoffe zu stützen, losgelöst von der Kenntnis ihrer Matrix. Die chemische Zusammensetzung wird über die Elemente und deren Speziierung ermittelt. Sie ist entscheidend für die Einordnung von Abfällen, deren Kenntnis ist aber auch eine notwendige Voraussetzung für die Beurteilung der stofflichen Qualitäten. Stimmen zwei Materialien in ihrer chemischen Zusammensetzung uiberein, so heißt das noch nicht, daß sie sich nach ihrer Ablagerung ähnlich verhalten in bezug auf Diagenese oder Verwitterung. Damit sie sich ähnlich verhalten, müssen sie auch in ihrer Phasenvergesellschaftung übereinstimmen, und es müssen die externen Faktoren gleich oder sehr ähnlich sein. Homogene Bereiche bei einem bestimmten Aggregatzustand (fest, flüssig, gasförmig) werden als Phasen bezeichnet ${ }^{1321}$. Unter homogenen Bereichen in Festkörpern sind sowohl Minerale als auch Gläser oder andere amorphe Festkörper mit jeweils gleichen Eigenschaften zu verstehen. An einem Kornaggregat (zum Beispiel Sediment) zählt man so viele Phasen, wie unterschiedliche Kornarten (Minerale, Glaspartikeln, 


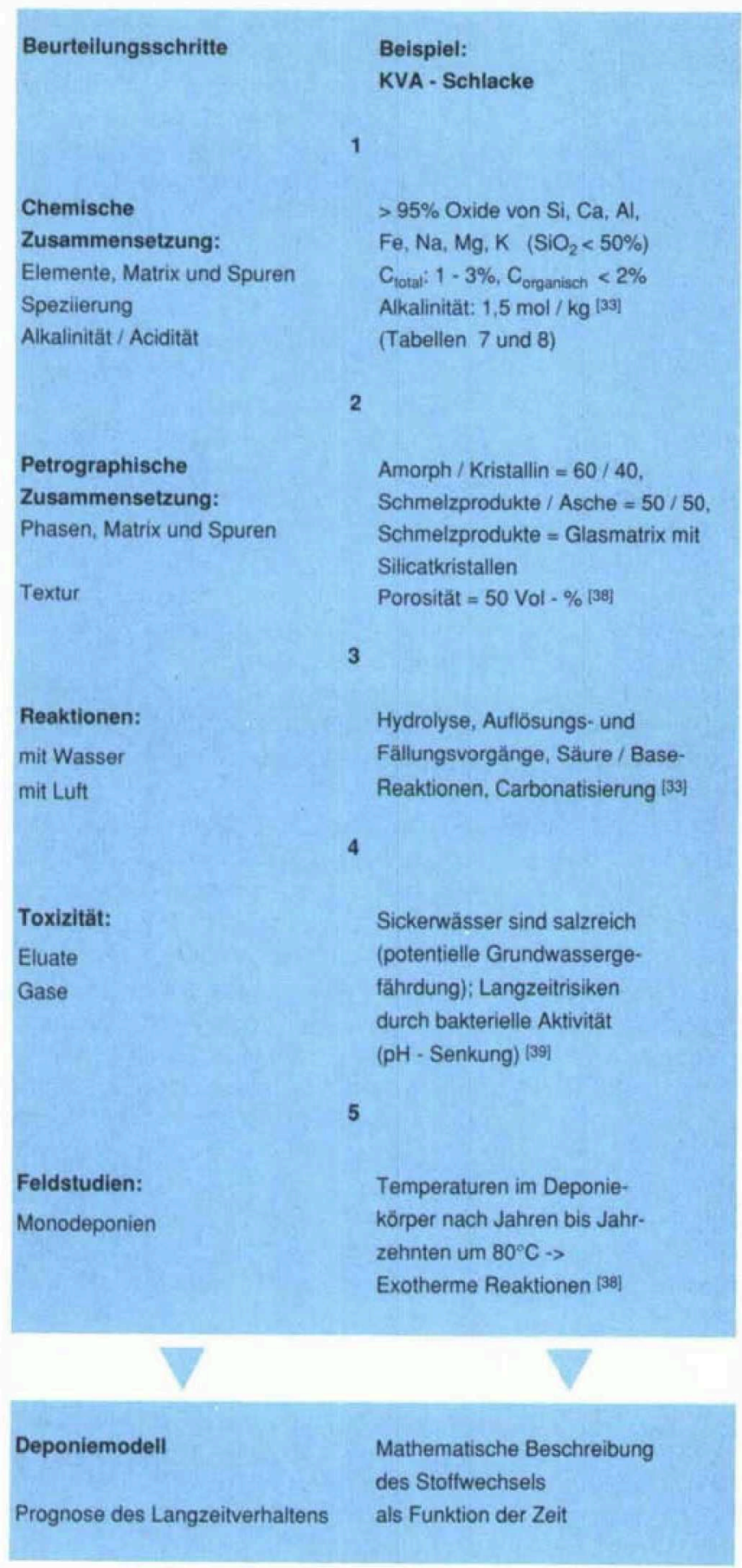

Figur 9. Schema der Beurteilung anthropogener Ablagerungen hinsichtlich ihrer Endlagerqualität. Am Beispiel der Schlacke aus einer Kehrichtverbrennungsanlage (KVA) wird der heutige Kenntnisstand illustriert. In allen Beurteilungsschritten ist das Verständnis noch lückenhaft. Eine mathematische Beschreibung des Stoffwechsels einer Schlackedeponie als Funktion der Zeit wird erst bei besseren Kenntnissen möglich sein.

etc.) darin vorkommen. In einem Gestein, das Olivin und Plagioklase (beides Silicatminerale) in einheitlicher glasiger Grundmasse enthält, bilden alle Olivinkristalle zusammen eine Phase, ebenso alle Plagioklase und auch das Glas; es liegen also drei Phasen vor. Als externe Faktoren sind etwa der Ablagerungsort (terrestrisch, lakustrisch, marin), das Klima und die Sedimentations- oder Erosionsraten in Betracht zu ziehen. Bei gleicher Sedimentzusammensetzung lassen sich verschiedene Szenarios entwerfen (Figur 8). Die identifizierten Phasen müssen einzeln und in ihrer Vergesellschaftung auf ihre Stabilität unter den Bedingungen der plausibelsten Szenarios geprüft werden. Die Erfahrungen aus den Erdwissenschaften, insbesondere aus der Petrographie, Thermodynamik und Geochemie bieten dafür die Basis. Diese Beurteilungen müssen unterstützt werden durch $\mathrm{Ex}$ perimente (Reaktion mit Wasser und/oder Luft in Laboratoriums und Feldexperimenten bis hin zu Deponieversuchen im Maßstab 1:1); die Emissionen (Eluate, Gase) sind hinsichtlich ihrer Toxizität für Mensch und Umwelt zu beurteilen. Schließlich werden die Ergebnisse dazu verwendet, ein mathematisches Modell (empirischer Ansatz) für den Stoff haushalt einer Deponie zu entwikkeln. Dieses Modell soll zuverlässigere Abschätzungen des stofflichen Langzeitverhaltens ermöglichen.

Die hier genannten Methoden zur Beurteilung eines für die Ablagerung vorgesehenen Materials sind schematisch in Figur 9 zusammengestellt und mit Ergebnissen aus Schlackenuntersuchungen illustriert ${ }^{133 !}$.

\subsection{Die Abschätzung des Deponiebedarfs}

\section{Triageorganisation und zugehörige Verfahrenstechnik}

In Zusammenarbeit mit der gesamten Volkswirtschaft sind ökonomische und soziologische Anreize zu schaffen und politische Entscheidungen zu treffen, damit wiederverwertbare Güter nicht mehr in den Abfall gelangen. Papier-, Glas- und Grünabfälle sowie sortenreine Produktionsabfälle dürfen nicht vermischt werden, sondern müssen separat erfaßt und in speziellen Anlagen für einen weiteren Gebrauch aufbereitet werden. Ebenfalls separat zu erfassen sind Sonderabfälle. Auch sie sind so wiederaufzubereiten, daß die Umweltbelastung dabei kleiner ist als die Belastung durch Entsorgung und Neuproduktion. Da dies nicht für alle Abfälle möglich sein wird, müssen parallel dazu Entsorgungsanlagen geschaffen werden, mit denen sich unter Einhaltung aller Emmissionsbeschränkungen und mit haushälterischem Energieaufwand Rückstandsqualitäten erreichen lassen, die der Forderung nach geringer Reaktivität genügen. Organische Sonderabfälle sind hierbei zu mineralisieren.

Die Baustellenabfälle aus Neu- und Umbauten sind möglichst sortenrein zu erfassen, so daß auch sie entweder zu Neumaterialien aufbereitet oder aber zumindest einer solchen Hinterlassenschaften angepaßten Entsorgung zugeführt werden können. Abbrüche müssen nach dem Prinzip des Rückbaus erfolgen, das heißt durch ein schrittweises Demontieren des Gebäudes. Dabei wird auf der Baustelle eine möglichst weitgehende Trennung in sortenreine Fraktionen möglich, die entweder eine Verwendung im gleichen Anwendungsbereich zuläßt (Beton soll wiederaufbereitet werden $\mathrm{zu}$ Recyclingbeton) oder aber eine maßgeschneiderte Aufbereitung zur Entsorgung. Für das dennoch verbleibende Bausperrgut müssen mechanische Sortieranlagen zur Verfügung stehen, die zumindest eine Abtrennung der thermisch verwertbaren Inhaltsstoffe gestatten.

\section{Aus- und Neubau von Entsorgungsanlagen}

In der als Modell gewählten Region "Metaland" entstehen bei der bisher üblichen Entsorgungspraxis jährlich ungefähr $900000 \mathrm{~m}^{3}\left(0.9 \mathrm{~m}^{3}\right.$ pro Einwohner) vermischte Bauabfälle. Durch gezielten Rückbau ist eine Abnahme auf $200000 \mathrm{~m}^{3} \mathrm{zu}$ erwarten (beim Rückbau verbleiben $18 \%$ als Bausperrgut oder Baumischabfall ${ }^{[34]}$ ). Dies entspricht dem jährlichen Durchsatz von zwei mechani- 
schen Sortieranlagen mit einer Jahreskapazität von rund $100000 \mathrm{~m}^{3}$. In den Anlagen werden Metalle und Brennbares abgetrennt. Die Metalle gehen in ein Schmelzwerk. Das Brennbare (50 Volumen-\%) wird thermisch verwertet (Müllverbrennungsanlage, Zementwerk oder Spezialofen). Die mineralische Fraktion $\left(100000 \mathrm{~m}^{3}\right)$ ist weiterzubehandeln oder in einer Reaktormonodeponie vom Typ $C_{A}$ abzulagern.

Der Klärschlamm wird zu $90 \%$ in Spezialöfen (zum Beispiel Wirbelschichtöfen) mineralisiert (Variante I). Mit einem Durchsatz von 6000 Tonnen pro Jahr (t/a) ${ }^{135 /}$ sind sechs Anlagen (oder eine Anlage pro 160000 Einwohner) erforderlich. Als feste Rückstände (Aschen und Neutralisationsschlämme) verbleiben pro Jahr rund 40000 Tonnen (circa $1 \mathrm{t}$ (feucht) / t TS, ${ }^{\mid 351}$ ). Die Aschen sind getrennt von den Neutralisationsschlämmen in Reaktormonodeponien vom Typ $C_{A}$ (Aschen) oder $C_{B}$ (Neutralisationsschlämme) abzulagern. Bei einer Dichte von 1.5 werden pro Jahr $30000 \mathrm{~m}^{3}$ (oder $0.03 \mathrm{~m}^{3}$ pro Einwohner) Deponieraum gebraucht (davon $3000 \mathrm{~m}^{3}$ als $C_{B}$ ). Wird der Klärschlamm in Schmelzöfen (Schmelzzyklon) behandelt (Variante II), so nimmt die zu deponierende Menge ab. Bei einem Durchsatz von 5 Tonnen Trockenschlamm pro Stunde wäre eine Großanlage erforderlich. Aus den 40000 Tonnen getrocknetem Klärschlamm würden jährlich ungefähr 20000 Tonnen glasige oder kristalline Schlacken hergestellt. Bei einer Dichte von 2.9 sind das rund $7000 \mathrm{~m}^{3}$. Falls die Schlacken nicht als Sekundärrohstoffe verwendbar sind, werden für Variante II jährlich $7000 \mathrm{~m}^{3}$ Deponieraum vom Typ $C_{A}$ benötigt. $\mathrm{Zu}$ sätzlich fallen etwa 1000 Tonnen Kondensate (Stäube) an. Sofern keine Verwendung möglich ist, müssen auch diese deponiert werden; dazu wären jährlich bis zu $1000 \mathrm{~m}^{3}$ Deponieraum vom Typ $C_{B}$ erforderlich.

Aus fünf Kehrichtverbrennungsanlagen (siehe Tabelle 1) sind jährlich 120000 Tonnen Kehrichtschlacken und 20000 Tonnen Rauchgasrückstände (Filterstäube und Rückstände der Feinreinigung) zu entsorgen. Werden die Schlacken nach Abtrennen der Grobeisenteile direkt in einer Reaktormonodeponie vom Typ $C_{A}$ abgelagert ( $\mathrm{Va}$ riante I), so sind dafür jährlich $85000 \mathrm{~m}^{3}$ (oder $0.09 \mathrm{~m}^{3}$ pro Einwohner) erforderlich (Dichte 1.4). Für die Ablagerung der Rauchgasreinigungsrückstände werden zusätzlich $20000 \mathrm{~m}^{3}$ (Subvariante Ia: Zugabe von hydraulischen Bindemitteln) oder bis zu $9000 \mathrm{~m}^{3}$ (Subvariante
Ib: Schmelzverfahren mit Schmelzprodukt und Kondensat) benötigt. Die Ablagerung der Rauchgasrückstände muß im Falle der Subvariante Ia in einer Monodeponie vom Typ $C_{B}$ erfolgen. In Subvariante Ib sind die Schmelzprodukte (glasartige oder kristalline Rückstände), falls nicht ats Sekundärrohstoff verwendbar, in eine Monodeponie vom Typ $C_{A}$ einzulagern. Die Kondensate ( 1500 $\mathrm{m}^{3}$ ) müssen, sofern nicht rezyklierbar, in einer Monodeponie vom Typ $C_{B}$ abgelagert werden.

Werden Schlacke und Rauchgasrückstände in Schmelzöfen (zum Beispiel Schmelzzyklon) nachbehandelt (Variante II), so braucht man wesentlich weniger Deponieraum; selbst dann, wenn eine Verwendung der Rückstände als Sekundärrohstoffe nicht oder noch nicht realisierbar sein sollte. Die in Kehrichtschlacken vorhandenen Poren (50 Volumen-\%) werden bis auf eine Restporosität von wenigen Prozent reduziert, wodurch sich die Rohdichte nahezu verdoppelt. Eine weitere Volumenreduktion wird durch die vollständige Mineralisierung (Zersetzung des organischen Materials) bewirkt. Die stoffliche Trennung in "gesteinsähnlich" (Deponietyp $A$, Schmelzprodukt) und "erzähnlich" (Deponietyp $B$, Kondensat) wird zudem verbessert. Im Falle der Deponierung sind für die glasigen oder kristallinen Schmelzprodukte jährlich maximal $35000 \mathrm{~m}^{3}$ vom Typ $C_{A}$ erforderlich. Für die Kondensate werden maximal 10000 $\mathrm{m}^{3}$ vom Typ $C_{B}$ benötigt. Da die Anwendungsmöglichkeiten für beide Produkte im Vergleich zu Variante I wesentlich verbessert sind, ist dieser Deponiebedarf als "worst case" zu betrachten.

In "Metaland" werden also jährlich für die Produkte aus der Behandlung von Bauabfällen, Siedlungsabfällen und Klärschlamm insgesamt rund $210000 \mathrm{~m}^{3}$ Deponieraum vom Typ $C_{4}\left(0.2 \mathrm{~m}^{3}\right.$ pro Einwohner) und $23000 \mathrm{~m}^{3}\left(0.02 \mathrm{~m}^{3}\right.$ pro Einwohner) Deponieraum vom Typ $C_{B}$ benötigt (Tabelle 9). Werden Hochtemperaturverfahren angewendet (auch für Schlacke und Klärschlamm), so beläuft sich der pro Jahr erforderliche Deponieraum auf rund $140000 \mathrm{~m}^{3}$ vom Тyp $C_{A}$ und rund $11000 \mathrm{~m}^{3}$ vom Typ $C_{B}$ (ohne Berücksichtigung der verbesserten Verwendbarkeit). Weggelassen wurden in dieser Abschätzung bisher die Shredderund Pneu- sowie die Sonderabfälle. Für sie werden jährlich maximal $10000 \mathrm{~m}^{3}$ benötigt $\left(C_{A}: C_{B}=10: 1\right)$.

Bei einer mittleren Deponiemächtigkeit von $10 \mathrm{~m}$ wird also im "Metaland" jährlich eine Fläche von $25000 \mathrm{~m}^{2}$ (oder $0.025 \mathrm{~m}^{2}$ pro Einwohner, Varianten I) respektive $16000 \mathrm{~m}^{2}$ (Varianten II) benötigt. Bei vollständiger Rezyklierung der Kondensate sinkt der Flächenbedarf in Variante II auf weniger als $15000 \mathrm{~m}^{2}$. Nimmt man zum Vergleich die bisher beobachteten Flächenbedürfnisse pro Einwohner und Jahr für die Erweiterung der Siedlungsfläche (Wohn- und Arbeitsplätze und Verkehrswege) von 2-3 $\mathrm{m}^{2}{ }^{\text {136! }}$, so ist der Deponieflächenbedarf (als Teil der Anthroposphäre) nur im Bereich von Prozenten. Begreift man hingegen diesen Materialflux als potentiellen Sedimentationsproze $\beta$, so entspricht das einer

Tabelle 9. Pro Jahr benötigtes Deponievolumen in der Modellregion "Metaland" (Fläche: $2500 \mathrm{~km}^{2}$, Bevölkerung: 1000 000). Für Variante II entspricht das einer Sedimentationsrate von $6 \mathrm{~cm}$ pro 1000 Jahre. Die Deponiemengen in Tonnen pro Einwohner und Jahr (letzte Zeile) addieren sich jährlich zu den im "Metaland" schon vorhandenen 40 t/E (Tabelie1). Variante I: Nach einer Mineralisierung in Verbrennungsanlagen. Variante II: Nach einer Aufschmelzung mittels Hochtemperaturverfahren.

\begin{tabular}{|c|c|c|c|c|}
\hline \multirow{2}{*}{$\begin{array}{l}\text { Varianten } \\
\text { Einheiten } \\
\text { Deponietypen }\end{array}$} & \multicolumn{2}{|c|}{$\begin{array}{c}1 \\
{\left[\mathrm{~m}^{3} / \mathrm{a}\right]}\end{array}$} & \multicolumn{2}{|c|}{$\stackrel{11}{\left[\mathrm{~m}^{3} / \mathrm{a}\right]}$} \\
\hline & $C_{A}$ & $\mathrm{C}_{\theta}$ & $C_{A}$ & $\mathrm{C}_{B}$ \\
\hline $\begin{array}{l}\text { Bauabfälle } \\
\text { mineralisch/nicht verwertbar }\end{array}$ & 100000 & & 100000 & \\
\hline $\begin{array}{l}\text { Klärschlammverbrennung } \\
\text { feste Rückstände }\end{array}$ & 27000 & 3000 & 7000 & 1000 \\
\hline $\begin{array}{l}\text { Kehrichtverbrennung } \\
\text { feste Rückstände }\end{array}$ & 85000 & 20000 & 35000 & 10000 \\
\hline $\begin{array}{l}\text { Deponievolumen total } \\
\text { (inklusive Shredder-/Pneu- } \\
\text { und Sonderabfälle) }\end{array}$ & $\begin{array}{l}212000 \\
(221000)\end{array}$ & $\begin{array}{l}23000 \\
(24000)\end{array}$ & 142000 & 11000 \\
\hline
\end{tabular}

Deponiemenge

pro Einwohner [t/a]

0.32

0.02

0.28

0.01

Anmerkung: Die bei Variante II eingesetzten Werte sind als "worst case" zu betrachten; dies insofern, als die verbesserten Verwendungschancen als Sekundärrohstoffe noch nicht berücksichtigt wurden. Es wurde von der pessimistischen Annahme ausgegangen, daß sämtliche festen Schmelzprodukte (inklusive Kondensate) zu deponieren sind. 
Sedimentationsrate von $6 \mathrm{~cm}$ pro 1000 Jahre, verteilt über die Fläche von ganz "Metaland", einer Rate also, die in der gleichen Größenordnung liegt wie eine mittlere natürliche Sedimentation. Das Postulat der Erdkrustenähnlichkeit wird deshalb sehr dringlich, es muissen Qualitäten erreicht werden, die sich ohne erhöhtes Risiko für die Umwelt direkt wieder verwenden lassen.

\section{Zusammenfassung und Schlußfolgerungen}

Die in der Einleitung gestellten Fragen können in knappster Form wie folgt beantwortet werden:

- Welche quantitative und qualitative Bedeutung haben die schon vorhandenen Deponien, unter denen sich auch die sogenannten Altasten befinden, für die zukünftige Ressourcenbewirtschaftung und die Umweltschutzstrategie der Region?

Eine auf lückenhaften Daten basierende erste grobe Abschätzung führt zum Schluß, daß die bestehenden Deponien mit wenigen Ausnahmen potentielle Altlasten sind, deren Emissionen noch von den nächsten Generationen behandelt werden müssen und von denen mit aller
Wahrscheinlichkeit ein gewichtiger Teil (mindestens $10 \%$ ) saniert werden muß. Kurzfristig sind ohne strenge Kontrollen lokale Grundwasser- und teilweise auch Oberflächenwasser-Verschmutzungen zu erwarten. Mittel- und langfristig muß aber ohne Sanierung mit einer regionalen Grundwasserverschmutzung gerechnet werden, vor allem durch organische Emissionen. Sanierung bedeutet hier die Summe von zusätzlichen bau- und verfahrenstechnischen Maßnahmen, um eine Umweltbelastung zu vermeiden. Die bis heute entstandenen anthropogenen Sedimente sind aus ökonomischer und ökologischer Sicht (mit wenigen Ausnahmen) keine potentiellen Ressourcen.

- Welche Konsequenzen ergeben sich für den Bau und den Betrieb der neu zu schaffenden Deponien und darüber hinaus für den ganzen Stoffhaushalt dieser Volkswirtschaft?

In einer ökologisch orientierten Volkswirtschaft, die das Vorsorgeprinzip konsequent anwenden will, muß die bisherige Praxis verlassen werden. Es müssen Monodeponien mit gesteinsähnlichen oder erzähnlichen Materialien geschaffen werden, die nicht nur umweltverträgliche Sedimente bilden (Endlagerqualität), sondern auch potentielle Ressourcen für nächste Generationen sein können. Der Flächenbedarf solcher Deponien ist, gemessen an der ganzen

\section{REAKTORDEPONIE}

Siedlungsfläche und deren kurzfristig noch zu erwartender Erweiterung, relativ klein. Das Deponieraumproblem für zukünftige Ablagerungen ist also raumplanerisch und technisch betrachtet ein qualitatives Problem, welches zuerst in der vorgeschalteten Behandlung und später schon in der Versorgung gelöst werden muß. Langfristig betrachtet ist jedoch die anthropogene Sedimentation in dicht besiedelten Regionen, gemessen an den geogenen Prozessen, ein quantitativ bedeutender Prozeß. Ein eigentlicher Paradigmawechsel in der Deponierung bedeutet somit kurzfristig zusätzliche und bessere Behandlungsanlagen in der Abfallwirtschaft und eine Anpassung der Prozesse und Güter in der Versorgung (Figur 10). Die Realisierung dieser Neuorientierung setzt eine Vertrauensbildung in der ganzen Bevölkerung voraus, und zwar deshalb, weil die heute bestehenden Deponien in den nächsten Jahren und Jahrzehnten als Altlasten das Exempel einer schlechten Ablagerung bleiben werden, das die $\mathrm{Zu}$ versicht in die bessere Entsorgung immer wieder erschüttern kann.

Figur 10. Schematische Darstellung des Übergangs vom Abfall zum Endlager. Bei der Abjallbehandlung werden Stoffe hergestellt oder separiert, die sich für eine Deponierung eignen. In der Reaktordeponie laufen geochemische und mikrobielle Prozesse $a b$, deren Produkte (Gase und Sickerwasser) noch behandelt werden müssen. Durch die Diagenese des Deponieinhalts wird schließlich Endlagerqualität erreicht.

\section{ENDLAGER}

\section{ABFALL}

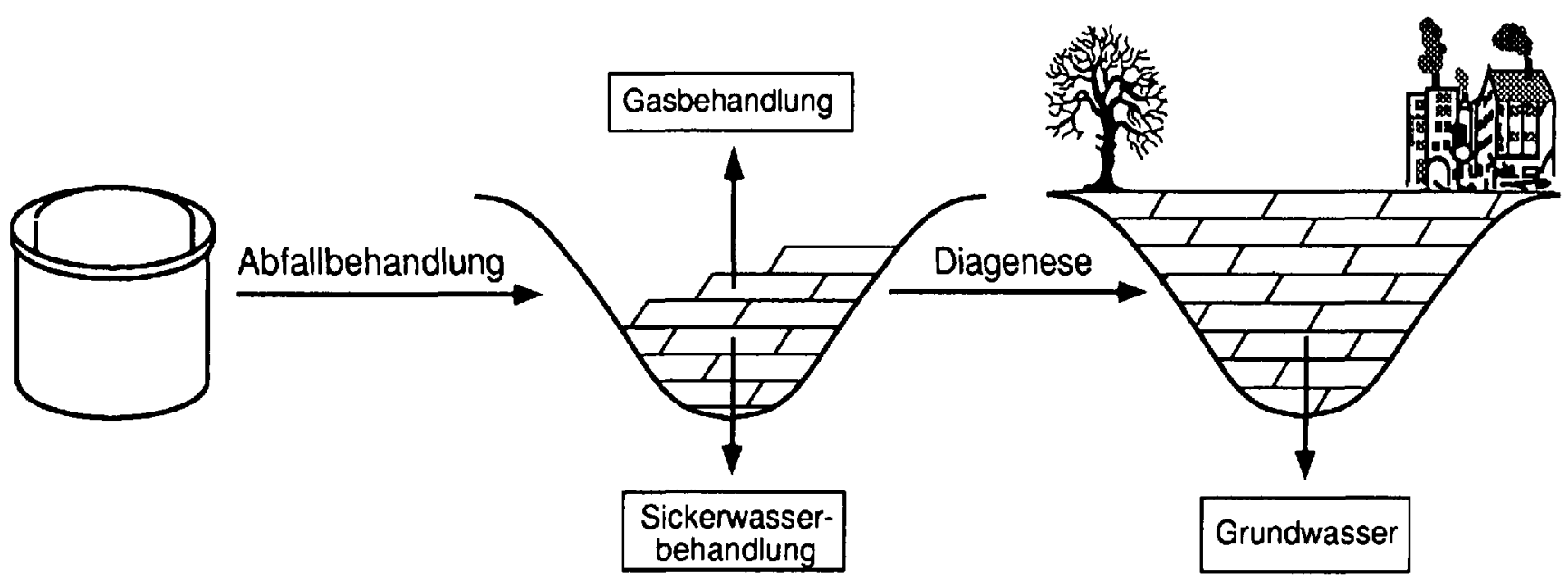


Daraus lassen sich für die Forschungspolitik allgemein und die Früherkennung folgende Schlußfolgerungen ableiten:

Die Entsorgungstrategien sind hinsichtlich ihrer Umsetzung sozialwissenschaftlich (inklusive Ökonomie) schlecht abgestützt. Es fehlt an empirischen Untersuchungen, welche den Ablauf bisheriger Entscheidungsprozesse kritisch evaluiert haben, um daraus allgemein gültige Zusammenhänge erkennen $\mathrm{zu}$ können. Es fehlt an kreativen politischen Verfahren, die zum Erreichen der angestrebten Ziele gebraucht werden. Bisher wurden Entscheidungsverfahren vor allem an neuen Bedürfnissen im Bildungs-, Sozial- und Wirtschaftsbereich gemessen, bei denen auf Motivationen für eine erlebbare Verbesserung im eigenen Umfeld bei einer Mehrheit der Bevölkerung gebaut werden konnte. Im Entsorgungsbereich hingegen wird bei der Realisierung von einer Minderheit eine Solidarität mit einem relativ großen Gemeinwesen verlangt, die - wie viele jüngste Beispiele zeigen - nicht mehr im klassischen Vollzugsverfahren erzwungen werden kann. Mit anderen Worten: Das ordnungspolitisch geprägte Instrumentarium des Umweltschutzes reicht nicht aus, um an das gesteckte Ziel zu gelangen.

Aus naturwissenschaftlich-technischer Sicht ist die ökologisch motivierte Endlagerqualität anthropogener Sedimente noch ungenügend charakterisiert. Diese Lücke kann und muß innert der nächsten zehn Jahren geschlossen werden, um das erwähnte politische Verfahren mit den notwendigen technischen Instrumenten zu unterstützen und in der Praxis die Verbesserung erlebbar zu machen.

In der Kombination dieser beiden Hauptaspekte eröffnet sich hier ein Feld partnerschaftlicher Projekte von Vollzugsbehörden, Privatwirtschaft und Hochschulforschung. Die unterschiedlichen Rollen dabei sind klar: Die Vollzugsbehörde (die Exekutive einer politisch souveränen Region) schafft den rechtlichen Rahmen, der die Verwirklichung einer neues Entsorgungskonzepts in ihrer Region möglich macht. Die Privatwirtschaft trägt das planerische Können bei und die verfahrenstechnische Entwicklung. Die Hochschule schließlich entwickelt die methodischen Instrumente zur Beurteilung der Szenarien. Für die neunziger Jahre bleibt zu prüfen, wie ein solches Pilotprojekt in transdisziplinärer Gestaltung zu inititieren und zu fördern wäre.

Diese Studie wurde vom Schweizerischen Wissenschaftsrat im Rahmen der forschungspolitischen Früherkennung (FER) finanziell unterstï̈zt.

\section{Literaturverzeichnis}

[1] P. Baccini, P. H. Brunner: Metabolism of the Anthroposphere, Springer-Verlag, Berlin (1991).

[2] P. Baccini, P. H. Brunner: "Der EinfluB von Maßnahmen auf den Stoffhaushalt der Schweiz, insbesondere auf die Entsorgung von Abfällen", Müll und Abfall (1990) Nr. 5 , 252-270.

[3] H. Wüest, C. Gabathuler: "Bauliche Ressourcen und ihre Benutzung, Lagebeurteilung und mögliche Verwendungspotentiale", in ETH-Forschungsprojekt MANTO, Spezialstudie 2.23, ETH Zürich (1985)

[4] H.-P. Fahrni: "Möglichkeiten und Grenzen yon Maßnahmen in der Abfallverminderung", Umweltschutz in der Schweiz 4 (1989) 12-20.

[5] G. Hösel: Unser Abfall aller Zeiten, Jehle, München (1987).

[6] H.-P. Lühr (Ed.): Die Deponie - ein Bauwerk?, IWS-Schriftenreihe $1 / 1987$, Institut für wassergefährdende Sıoffe, Technische Universität Berlin (1987).

[7] H. Belevi, P. Baccini: "Long-Term Behavior of Municipal Solid Waste Landfills", Waste Management \& Research 7 (1989) 43-56.

[8] D. Fischer, W. Schenkel: "Anforderungen an die Ablagerungen von Abfällen in der Bundesrepublik Deutschland, der Schweiz und Österreich", Müll und Abfall (1990) Nr. 1, 2-13.

[9] Eidgenössische Kommission für Abfallwirtschaft: Leibild für die schweizerische Abfallwirtschaft, Schriftenreihe Umweltschutz Nr. 5I, BUWAL, Bern (1986).

[10] P. Baccini (Ed.): The Landfill: Reactor and Final Storage, Lecture Notes in Earth Sciences, Vol. 20, Springer-Verlag, Berlin (1989)

[11] H. Jonas: Das Prinzip Verantwortung. Versuch einer Ethik für die technologische Zivilisation, Insel, Frankfurt am Main (1979).

[12] H.-P. Müller: "Praktische Probleme in der Erfassung und Behandlung von Altlasten", Pro Aqua - Pro Vira, 10. Intemationale Fachmesse und Fachtagungungen für Umweltschutztechnik und Ökologie, 0ktober 1986, Vol. D, 4.1-4.4.20, Basel (1986)

[13] W. Gujer: "Die Entwicklung des Klärschlammanfalles in der Schweiz", Mitteilungen der EAWAG 28 (1989) 2-5.

[14] P. Baccini, M. Baechler, P. H. Brunner, G. Henseler: "Von der Entsorgung zum Stoffhaushalt. Die Steuerung anthropogener Stoffflüsse als interdisziplinäre Aufgabe", Müll und Abfall (1985) Nr. 4, 99-108.

[15] P. Baccini, P. H. Brunner: "Behandlung und Endlagerung von Rückständen aus der Kehrichtverbrennung", Gas, Wasser Abwasser 65 (1985) 403-409.

[16] Amt für Gewässerschutz und Wasserbau des Kantons Zürich ( $\mathrm{AGW}$ ): Entsorgung von Baurestmassen im Kanton Zürich, Zürich (1990).

[17] Amt für Gewässerschutz und Wasserbau des Kantons Zürich (AGW): Zahlen zur Abfallwirtschafi im Kanton Zürich 1989. Zürich (1990).

[18] P. Baccini, R. Zimmerli, M. Krähenbüh]: "Bestimmung und Beurteilung der physikalisch-chemischen Eigenschaften von Abfallen der Autoverwertung Ostschweiz AG", EAWAG-Projekt Nr. 30-323, Dübendorf (1985).

[19] Schweizcrischer Bundesrat: Verordmung über den Verkehr mit Sonderabfällen (VVS) Bern (1985).
[20] P. Baccini, P. H. Brunner: "Long-Term Implications of the Land Disposal of Residuals from the Management of Municipal and industrial Solid Wastes", Proceedings of the I0th Ammual Madison Waste Conference, Department of Engineering Professional Development, University of Wisconsin, Madison WI (1987)

[2I] P. Baccini, G. Henseler, R. Figi, H. Belevi: "Water and Element Balances of Municipal Solid Waste Landfills", Waste Management \& Research 5 (1987) 483-499.

[22] H. Belevi: "Stoffifuisse durch Reaktordeponien für Siedlungsabfalle", Abfallspektrum 3/2 (1991) 16-21.

[23] Schweizerischer Bundesrat: Verordinung über Abwassereinleitungen, Bern (1975).

[24] H. Bclevi, P. Baccini: "Water and Elemen Fluxes from Sanitary Landfills"; in T. H. Christensen, R. Cossu, R. Stegmann (Ed.): Sanirary Landfilling: Process, Technology and Environmental Impact, Academic Press, London (1989)

[25] H. Belevi, P. Baccini: "Long-Term Assessment of Leachates from Municipal Solid Waste Landfills", Proceedings of the 2nd Inernational Landfill Symposium, ISWA, Porto Conte, ltaly (October 1989).

[26] V. Franzius, R. Stegmann, K. Wolf: Handbuch der Altlastensanierung, R. von Deckers, Heidelberg (1988).

[27] F. W. Clarke: "The Data of Geochemistry", Bulletin of the U.S. Geological Surveillance, No. 770 (1924) 841.

[28] W. H. Ernst: Schwermetallvegetation der Erde, Gustav Fischer, Stuttgart (1974).

[29] K. K.Turekian: Chemistry of the Earth, Physical Science and Technology Series, Holt, Rinehart and Winston, New York (1972).

[30] P. Ramdohr, H. Strunz: Klockmanns Lehrbuch der Mineralogie, 16.Auflage, Enke, Stultgart (1978).

[31] Schweizerischer Bundesrat: Technische Verordnung über Abfälle (TVA), Bern (1990)

[32] E. Nickel: Grundwissen in Mineralogie, Teil 3: Aufbaukursus Petrographie, Ott, Thun (1975).

[33] H. Belevi, D. Stämpfli, P. Baccini: "Methods to Assess the Chemical Bchavior of Municipal Solid Waste Incinerator Bottom Ash in Monofills", Waste Management \& Research (1991), submilted for publication.

[34] P. Staub: "Der schnelle Abbruch schont weder Umwelt noch Portemonnaie", in Der Unternehmer im Spannungsfeld Versorgung - Entsorgung, Tagungsbericht der Schweizerischen Interessengemeinschaft für Abfallverminderung (SIGA), Zürich (1989).

[35] A. Obrist: "Technik der Klärschlammverbrennung", Mittcilungen der EAWAG (1989) 28.

[36] P. A. Rumley: Evolution passée et schémas prospectifs de l'utilisation du sol en Suisse, ORL-Bericht Nr. 50, ETH Zürich (1984).

[37] H. J. M. Bowen: Environmental Chemistry of the Elements, Academic Press, London (1979).

[38] T. Lichtensteiger: "Kehrichtschlacken als anthropogene Sedimente", EAWAG Jahresbericht 1990, Dübendorf (1991).

[39] N. Agustoni, H. Belevi, Z. Egli, P. Baccini: "Mikrobieller Abbau yon organischen Komponenten in KVA-Schlacken", EAWAG Jahresbericht 1990, Dübendorf (1991). 